\title{
Strong Factorizations of Operators with Applications to Fourier and Cesàro Transforms
}

\author{
O. Delgado, M. Mastyło, \& E. A. Sánchez Pérez
}

\begin{abstract}
Consider two continuous linear operators $T: X_{1}(\mu) \rightarrow$ $Y_{1}(v)$ and $S: X_{2}(\mu) \rightarrow Y_{2}(\nu)$ between Banach function spaces related to different $\sigma$-finite measures $\mu$ and $\nu$. By means of weighted norm inequalities we characterize when $T$ can be strongly factored through $S$, that is, when there exist functions $g$ and $h$ such that $T(f)=$ $g S(h f)$ for all $f \in X_{1}(\mu)$. For the case of spaces with Schauder basis, our characterization can be improved, as we show when $S$ is, for instance, the Fourier or Cesàro operator. Our aim is to study the case where the map $T$ is besides injective. Then we say that it is a representing operator-in the sense that it allows us to represent each element of the Banach function space $X(\mu)$ by a sequence of generalized Fourier coefficients-providing a complete characterization of these maps in terms of weighted norm inequalities. We also provide some examples and applications involving recent results on the Hausdorff-Young and the Hardy-Littlewood inequalities for operators on weighted Banach function spaces.
\end{abstract}

\section{Introduction}

Let $X_{1}(\mu), X_{2}(\mu), Y_{1}(\nu), Y_{2}(\nu)$ be Banach function spaces related to different $\sigma$-finite measures $\mu$ and $\nu$. We consider two continuous linear operators $T: X_{1}(\mu) \rightarrow Y_{1}(\nu)$ and $S: X_{2}(\mu) \rightarrow Y_{2}(\nu)$. In this paper, in terms of weighted norm inequalities, we provide a characterization of when $T$ can be factored through $S$ via multiplication operators, that is, when there are functions $g$ and $h$ satisfying that $T(f)=g S(h f)$ for all $f \in X_{1}(\mu)$.

This problem was studied in [6] for the case where $\mu$ and $v$ are the same finite measure. However, the results developed there do not allow us to face the problem we study here, in which different $\sigma$-finite measures $\mu$ and $\nu$ appear in order to consider the relevant case of the classical sequence spaces $\ell^{p}$. The reason is that we are interested in considering standard cases as the Fourier and Cesàro operators, which will be in fact our main examples.

Received March 27, 2017. Revision received September 14, 2018.

The first author gratefully acknowledge the support of the Ministerio de Economía y Competitividad (project \#MTM2015-65888-C4-1-P) and the Junta de Andalucía (project FQM-7276), Spain. The second author was supported by National Science Centre, Poland, project no. 2015/17/B/ST1/00064. The third author acknowledges with thanks the support of the Ministerio de Economía y Competitividad (project MTM2016-77054-C2-1-P), Spain. 
In this direction, we will show that in the case where the Köthe dual $Y_{1}(v)^{\prime}$ of $Y_{1}(v)$ and $X_{1}(\mu)$ have a Schauder basis the norm inequality that characterizes the factorization of $T$ through $S$ can be weakened. After showing this, we will develop with some detail some examples regarding Fourier operators, operators factoring though infinite matrices, and the Cesàro operator. This will allow us to introduce the notion of representing operator and to study it in the second part of the paper.

Let us explain briefly this notion. With the notation introduced, assume that $Y_{1}(v)$ and $Y_{2}(v)$ have unconditional bases $\mathcal{U}_{1}:=\left\{v_{i}: i \in \mathbb{N}\right\}$ and $\mathcal{U}_{2}:=\left\{e_{i}: i \in\right.$ $\mathbb{N}\}$, respectively. Suppose that there exists a Schauder basis $\mathcal{B}:=\left\{f_{i}: i \in \mathbb{N}\right\}$ for the space $X_{2}(\mu)$ and write $\alpha_{i}(f)$ for the $i$ th basic coefficient of $f \in X_{2}(\mu), i \in \mathbb{N}$. We will say that an operator $T: X_{1}(\mu) \rightarrow Y_{1}(v)$ is a representing operator (associated with the basis $\mathcal{B}$ of $\left.X_{2}(\mu)\right)$ if each element $x \in X_{1}(\mu)$ can be represented univocally by a sequence of coefficients $\left(\beta_{i}(x)\right)$ such that $\sum_{i=1}^{\infty} \beta_{i}(x) v_{i} \in Y_{1}(v)$, where the coefficients $\beta_{i}(x)$ can be computed by means of the associated values of $\alpha_{i}$ by a simple transformation provided by multiplication operators.

Thus the last part of the paper is devoted to find a characterization of such operators in terms of vector norm inequalities they must satisfy. We provide also classical and recently published examples of such kind of maps, using, for instance, an improvement of the Hausdorff-Young inequality given in [8] or the continuity of the Fourier operator $\mathcal{H}_{p}: L^{p}[-\pi, \pi] \rightarrow \ell^{p}(W)$, where $\ell^{p}(W)$ is a weighted $\ell^{p}$-space, which can be found in [1].

\section{Preliminaries}

Let $(\Omega, \Sigma, \mu)$ be a $\sigma$-finite measure space and denote by $L^{0}(\mu)$ the space of all measurable real functions defined on $\Omega$, where functions that are equal $\mu$-a.e. are identified. By a Banach function space we mean a Banach space $X(\mu) \subset L^{0}(\mu)$ with norm $\|\cdot\|_{X}$ such that if $f \in L^{0}(\mu), g \in X(\mu)$, and $|f| \leq|g| \mu$-a.e., then $f \in X(\mu)$ and $\|f\|_{X} \leq\|g\|_{X}$. In particular, $X(\mu)$ is a Banach lattice for the $\mu$ a.e. pointwise order, in which the convergence in norm of a sequence implies the convergence $\mu$-a.e. for some subsequence. Note that every positive linear operator between Banach lattices is continuous, (see [11, p. 2]). So, all inclusions between Banach function spaces are continuous. General information about Banach function spaces can be found, for instance, in [18, Ch. 15], considering the function norm $\rho$ defined there as $\rho(f)=\|f\|_{X}$ if $f \in X(\mu)$ and $\rho(f)=\infty$ otherwise.

A Banach function space $X(\mu)$ is said to be saturated if there is no $A \in \Sigma$ with $\mu(A)>0$ such that $f \chi_{A}=0 \mu$-a.e. for all $f \in X(\mu)$. This is equivalent to the existence of a function $g \in X(\mu)$ such that $g>0 \mu$-a.e.

Given two Banach function spaces $X(\mu)$ and $Y(\mu)$, the $Y(\mu)$-dual space of $X(\mu)$ is defined by

$$
X^{Y}=\left\{h \in L^{0}(\mu): f h \in Y(\mu) \text { for all } f \in X(\mu)\right\} .
$$

Every $h \in X^{Y}$ defines a continuous multiplication operator $M_{h}: X(\mu) \rightarrow Y(\mu)$ via $M_{h}(f)=f h$ for all $f \in X(\mu)$. The space $X^{Y}$ is a Banach function space with 
norm

$$
\|h\|_{X^{Y}}=\sup _{f \in B_{X}}\|h f\|_{Y}, \quad h \in X^{Y},
$$

if and only if $X(\mu)$ is saturated ([12]). As usual, $B_{X}$ denotes the closed unit ball of $X(\mu)$. If $X(\mu)$ is saturated, then $X^{L^{1}}$ is just the classical Köthe dual space $X(\mu)^{\prime}$ of $X(\mu)$, and $X(\mu)^{\prime}$ is also saturated. This does not hold in general for $X^{Y}$. For issues related to generalized dual spaces, see [3] and the references therein.

A saturated Banach function space $X(\mu)$ is contained in its Köthe bidual $X(\mu)^{\prime \prime}$ with $\|f\|_{X^{\prime \prime}} \leq\|f\|_{X}$ for all $f \in X(\mu)$. It is known that $\|f\|_{X^{\prime \prime}}=\|f\|_{X}$ for all $f \in X(\mu)$ if and only if $X(\mu)$ is order semicontinuous, that is, if for any $f, f_{n} \in X(\mu)$ such that $0 \leq f_{n} \uparrow f \mu$-a.e., it follows that $\left\|f_{n}\right\|_{X} \uparrow\|f\|_{X}$. Even more, $X(\mu)=X(\mu)^{\prime \prime}$ with equal norms if and only if $X(\mu)$ has the $F a$ tou property, that is, if for any $f_{n} \in X(\mu)$ such that $0 \leq f_{n} \uparrow f \mu$-a.e. and $\sup _{n}\left\|f_{n}\right\|_{X}<\infty$, we have that $f \in X(\mu)$ and $\left\|f_{n}\right\|_{X} \uparrow\|f\|_{X}$.

Denote by $X(\mu)^{*}$ the topological dual of a saturated Banach function space $X(\mu)$. Every function $h \in X(\mu)^{\prime}$ defines an element $\eta(h) \in X(\mu)^{*}$ via $\langle\eta(h), f\rangle=\int h f d v$ for all $f \in X(\mu)$. The map $\eta: X(\mu)^{\prime} \rightarrow X(\mu)^{*}$ is a continuous linear injection, since the norm of every $h \in X(\mu)^{\prime}$ can be computed as

$$
\|h\|_{X^{\prime}}=\sup _{f \in B_{X}}\left|\int h f d \mu\right|,
$$

and so $\eta$ is an isometry. It is known that $\eta$ is surjective if and only if $X(\mu)$ is $\sigma$-order continuous, that is, if for every $\left(f_{n}\right) \subset X(\mu)$ with $f_{n} \downarrow 0 \mu$-a.e. it follows

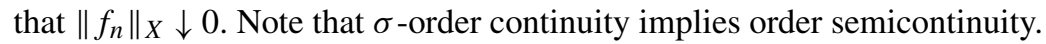

The $\sigma$-order continuous part $X_{a}(\mu)$ of a saturated Banach function space $X(\mu)$ is the largest $\sigma$-order continuous closed solid subspace of $X(\mu)$ that can be described as

$$
X_{a}(\mu)=\left\{f \in X(\mu):|f| \geq f_{n} \downarrow 0 \mu \text {-a.e. implies }\left\|f_{n}\right\|_{X} \downarrow 0\right\} .
$$

Also, a function $f \in X_{a}(\mu)$ if and only if $f \in X(\mu)$ satisfies that $\left\|f \chi_{A_{n}}\right\|_{X} \downarrow 0$ whenever $\left(A_{n}\right) \subset \Sigma$ is such that $A_{n} \downarrow$ with $\mu\left(\bigcap A_{n}\right)=0$. Note that $X_{a}(\mu)$ can be the trivial space as in the case of $X(\mu)=L^{\infty}(\mu)$ when $\mu$ is nonatomic. It is always a Banach space. In the case where $X_{a}(\mu)$ is saturated, $X_{a}(\mu)$ is order dense in $L^{0}(\mu)$, and so by the monotone convergence theorem it follows easily that $X_{a}(\mu)^{\prime}=X(\mu)^{\prime}$ with equal norms.

The $\pi$-product space $X \pi Y$ of two Banach function spaces $X(\mu)$ and $Y(\mu)$ is defined as the space of functions $h \in L^{0}(\mu)$ such that $|h| \leq \sum_{n}\left|f_{n} g_{n}\right| \mu$-a.e. for some sequences $\left(f_{n}\right) \subset X(\mu)$ and $\left(g_{n}\right) \subset Y(\mu)$ satisfying $\sum_{n}\left\|f_{n}\right\|_{X}\left\|g_{n}\right\|_{Y}<\infty$. For $h \in X \pi Y$, consider the subadditive and homogeneous functional

$$
\pi(h)=\inf \left\{\sum_{n}\left\|f_{n}\right\|_{X}\left\|g_{n}\right\|_{Y}\right\},
$$

where the infimum is taken over all sequences $\left(f_{n}\right) \subset X(\mu)$ and $\left(g_{n}\right) \subset Y(\mu)$ such that $|h| \leq \sum_{n}\left|f_{n} g_{n}\right| \mu$-a.e. and $\sum_{n}\left\|f_{n}\right\|_{X}\left\|g_{n}\right\|_{Y}<\infty$ (see [17, Prop. 1.4] but notice that the functional defined there considers only finite sums). The space 
$X \pi Y$ is a saturated Banach function space with norm $\pi$ if and only if $X(\mu)$, $Y(\mu)$, and $X^{Y^{\prime}}$ are saturated, and in this case, $(X \pi Y)^{\prime}=X^{Y^{\prime}}$ with equal norms (see [5, Prop. 2.2]). The calculus of product spaces is nowadays well known (see $[3 ; 5 ; 10 ; 17])$; the reader can find all the information needed on this construction in these papers.

Banach function spaces on the measure space $(\mathbb{N}, \mathcal{P}(\mathbb{N}), \lambda)$ with counting measure $\lambda$ are usually called Banach sequence spaces. The classical Banach sequence space $\ell^{p}$ for $1 \leq p \leq \infty$ is saturated and is $\sigma$-order continuous if and only if $p<\infty$. As usual, for each $n \in \mathbb{N}$, we denote by $\left(e^{n}\right)$ the standard unit vector basis in $c_{0}$.

We recall the well-known easily verified formula $\left(\ell^{p}\right)^{\ell^{q}}=\ell^{s_{p q}}$ with equal norms, where

$$
1 \leq s_{p q}= \begin{cases}\frac{p q}{p-q} & \text { if } 1 \leq q<p<\infty, \\ q & \text { if } 1 \leq q<p=\infty, \\ \infty & \text { if } 1 \leq p \leq q \leq \infty .\end{cases}
$$

In particular, $\left(\ell^{p}\right)^{\prime}=\left(\ell^{p}\right)^{\ell^{1}}=\ell^{p^{\prime}}$, where $p^{\prime}$ denote the conjugate exponent of $p$ $\left(\frac{1}{p}+\frac{1}{p^{\prime}}=1\right)$. Note that $\ell^{p}$ has the Fatou property as $\left(\ell^{p}\right)^{\prime \prime}=\ell^{p}$. Also note that $s_{p q}=1$ if and only if $q=1$ and $p=\infty$.

\section{Strong Factorization of Operators on Banach Function Spaces}

Let $(\Omega, \Sigma, \mu),(\Delta, \Gamma, \nu)$ be $\sigma$-finite measure spaces, let $X_{1}(\mu), X_{2}(\mu), Y_{1}(\nu)$, $Y_{2}(\nu)$ be saturated Banach function spaces, and let $T: X_{1}(\mu) \rightarrow Y_{1}(v)$, $S: X_{2}(\mu) \rightarrow Y_{2}(v)$ be nontrivial continuous linear operators. For $h \in X_{1}^{X_{2}}$, we say that $T$ factors strongly through $S$ and $M_{h}$ if there exists $g \in Y_{2}^{Y_{1}^{\prime \prime}}$ such that the diagram

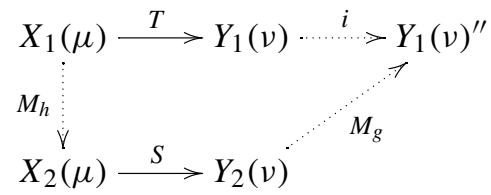

commutes. Here $i$ denotes the inclusion map. Note that if $Y_{1}(v)$ has the Fatou property, then this diagram looks as

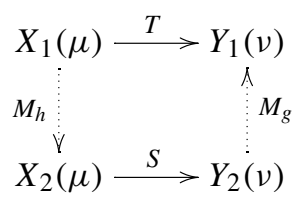

In the case where $\mu$ and $\nu$ are the same finite measures and under certain extra conditions, [6, Thm. 4.1] characterizes when $T$ factors strongly through $S$ and $M_{h}$. In this section, we extend this theorem to our more general setting and improve it by relaxing the conditions. The extension will be obtained from the following broader factorization result. 
THeOREM 3.1. Assume that $Y_{2}^{Y_{1}^{\prime \prime}}$ is saturated and consider a function $h \in X_{1}^{X_{2}}$. The following statements are equivalent:

(a) There exists a constant $C>0$ such that

$$
\sum_{i=1}^{n} \int T\left(x_{i}\right) y_{i}^{\prime} d v \leq C\left\|\sum_{i=1}^{n} S\left(h x_{i}\right) y_{i}^{\prime}\right\|_{Y_{2} \pi Y_{1}^{\prime}}
$$

for all $n \in \mathbb{N}, x_{1}, \ldots, x_{n} \in X_{1}(\mu)$, and $y_{1}^{\prime}, \ldots, y_{n}^{\prime} \in Y_{1}(\nu)^{\prime}$.

(b) There exists $\xi^{*} \in\left(Y_{2} \pi Y_{1}^{\prime}\right)^{*}$ satisfying the following factorization between the operators $T$ and $S$ :

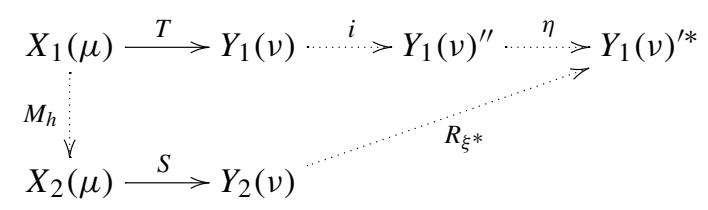

where $\eta$ is the continuous linear injection of $Y_{1}(v)^{\prime \prime}$ into $Y_{1}(v)^{\prime *}$, and $R_{\xi *}$ is the continuous linear operator defined by $\left\langle R_{\xi^{*}}\left(y_{2}\right), y_{1}^{\prime}\right\rangle=\left\langle\xi^{*}, y_{2} y_{1}^{\prime}\right\rangle$ for $y_{2} \in Y_{2}(v)$ and $y_{1}^{\prime} \in Y_{1}(v)^{\prime}$.

Proof. Note that the condition of $Y_{2}^{Y_{1}^{\prime \prime}}$ being saturated assures that $Y_{2} \pi Y_{1}^{\prime}$ is a saturated Banach function space. Also note that the map $R_{\xi^{*}}: Y_{2}(v) \rightarrow Y_{1}(v)^{\prime *}$ defined in (b) is a well-defined continuous linear operator as

$$
\left|\left\langle R_{\xi *}\left(y_{2}\right), y_{1}^{\prime}\right\rangle\right| \leq\left\|\xi^{*}\right\|_{\left(Y_{2} \pi Y_{1}^{\prime}\right) *}\left\|y_{2} y_{1}^{\prime}\right\|_{Y_{2} \pi Y_{1}^{\prime}} \leq\left\|\xi^{*}\right\|_{\left(Y_{2} \pi Y_{1}^{\prime}\right) *}\left\|y_{2}\right\|_{Y_{2}}\left\|y_{1}^{\prime}\right\|_{Y_{1}^{\prime}}
$$

for all $y_{2} \in Y_{2}(v)$ and $y_{1}^{\prime} \in Y_{1}(v)^{\prime}$.

(a) $\Rightarrow$ (b) For any $n \in \mathbb{N}, x_{1}, \ldots, x_{n} \in X_{1}(\mu)$, and $y_{1}^{\prime}, \ldots, y_{n}^{\prime} \in Y_{1}(v)^{\prime}$, we take the convex function $\phi: B_{\left(Y_{2} \pi Y_{1}^{\prime}\right)^{*}} \rightarrow \mathbb{R}$ given by

$$
\phi\left(\xi^{*}\right)=\sum_{i=1}^{n} \int T\left(x_{i}\right) y_{i}^{\prime} d v-C \sum_{i=1}^{n}\left\langle\xi^{*}, S\left(h x_{i}\right) y_{i}^{\prime}\right\rangle
$$

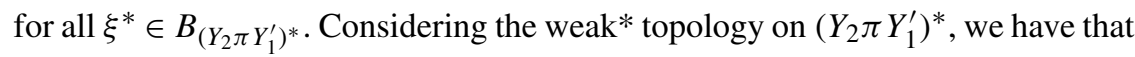
$\phi$ is a continuous map on a compact convex set. Moreover, by the Hahn-Banach theorem there exists $\xi_{\phi}^{*} \in B_{\left(Y_{2} \pi Y_{1}^{\prime}\right) * \text { such that }}$

$$
\left\|\sum_{i=1}^{n} S\left(h x_{i}\right) y_{i}^{\prime}\right\|_{Y_{2} \pi Y_{1}^{\prime}}=\left\langle\xi_{\phi}^{*}, \sum_{i=1}^{n} S\left(h x_{i}\right) y_{i}^{\prime}\right\rangle,
$$

and so by (a) it follows that $\phi\left(\xi_{\phi}^{*}\right) \leq 0$.

Since the family $\mathcal{F}$ of functions $\phi$ defined in this way is concave, Ky Fan's lemma (see, e.g., [15, E. 4]) guarantees the existence of an element $\xi^{*} \in B_{\left(Y_{2} \pi Y_{1}^{\prime}\right)^{*}}$ such that $\phi\left(\xi^{*}\right) \leq 0$ for all $\phi \in \mathcal{F}$. In particular, for all $x \in X_{1}(\mu)$ and $y^{\prime} \in Y_{1}(\nu)^{\prime}$, we have that

$$
\int T(x) y^{\prime} d v \leq C\left\langle\xi^{*}, S(h x) y^{\prime}\right\rangle .
$$


By taking $-y^{\prime}$ instead of $y^{\prime}$ we obtain that

$$
-\int T(x) y^{\prime} d v \leq-C\left\langle\xi^{*}, S(h x) y^{\prime}\right\rangle
$$

and so

$$
\left\langle\eta(T(x)), y^{\prime}\right\rangle=\left\langle R_{C \xi *}(S(h x)), y^{\prime}\right\rangle .
$$

Therefore $\eta(T(x))=R_{C \xi^{*}}(S(h x))$ for all $x \in X_{1}(\mu)$, and the factorization in (b) holds for $C \xi^{*} \in\left(Y_{2} \pi Y_{1}^{\prime}\right)^{*}$.

(b) $\Rightarrow$ (a) For each $n \in \mathbb{N}$ and all $x_{1}, \ldots, x_{n} \in X_{1}(\mu)$ and $y_{1}^{\prime}, \ldots, y_{n}^{\prime} \in Y_{1}(v)^{\prime}$, we have that

$$
\begin{aligned}
\sum_{i=1}^{n} \int T\left(x_{i}\right) y_{i}^{\prime} d v & =\sum_{i=1}^{n}\left\langle\eta\left(T\left(x_{i}\right)\right), y_{i}^{\prime}\right\rangle=\sum_{i=1}^{n}\left\langle R_{\xi *}\left(S\left(h x_{i}\right)\right), y_{i}^{\prime}\right\rangle \\
& =\sum_{i=1}^{n}\left\langle\xi^{*}, S\left(h x_{i}\right) y_{i}^{\prime}\right\rangle=\left\langle\xi^{*}, \sum_{i=1}^{n} S\left(h x_{i}\right) y_{i}^{\prime}\right\rangle \\
& \leq\left\|\xi^{*}\right\|_{\left(Y_{2} \pi Y_{1}^{\prime}\right)^{*}}\left\|\sum_{i=1}^{n} S\left(h x_{i}\right) y_{i}^{\prime}\right\|_{Y_{2} \pi Y_{1}^{\prime}} .
\end{aligned}
$$

Note that $\left\|\xi^{*}\right\|_{\left(Y_{2} \pi Y_{1}^{\prime}\right)^{*}}>0$ as $T$ is nontrivial.

Note that the condition of $Y_{2}^{Y_{1}^{\prime \prime}}$ being saturated is obtained, for instance, if $Y_{2}(v) \subset Y_{1}(v)^{\prime \prime}$, which is equivalent to $L^{\infty}(v) \subset Y_{2}^{Y_{1}^{\prime \prime}}$. Also note that condition (a) of Theorem 3.1 is equivalent to

$$
\left|\sum_{i=1}^{n} \int T\left(x_{i}\right) y_{i}^{\prime} d v\right| \leq C\left\|\sum_{i=1}^{n} S\left(h x_{i}\right) y_{i}^{\prime}\right\|_{Y_{2} \pi Y_{1}^{\prime}}, \quad n \in \mathbb{N},
$$

for all $x_{1}, \ldots, x_{n} \in X_{1}(\mu)$ and $y_{1}^{\prime}, \ldots, y_{n}^{\prime} \in Y_{1}(\nu)^{\prime}$. Indeed, we only have to take $-y_{1}^{\prime}, \ldots,-y_{n}^{\prime}$ instead of $y_{1}^{\prime}, \ldots, y_{n}^{\prime}$ in Theorem 3.1(a).

As a consequence of Theorem 3.1, we obtain the following generalization and improvement of [6, Thm. 4.1].

COROLlary 3.2. Assume that $Y_{2}^{Y_{1}^{\prime \prime}}$ is saturated and that $y_{2} y_{1}^{\prime} \in\left(Y_{2} \pi Y_{1}^{\prime}\right)_{a}$ for all $y_{2} \in Y_{2}(v)$ and $y_{1}^{\prime} \in Y_{1}(v)^{\prime}$. Given $h \in X_{1}^{X_{2}}$, the following statements are equivalent:

(a) The operator $T$ factors strongly through $S$ and $M_{h}$.

(b) There exists a constant $C>0$ such that

$$
\sum_{i=1}^{n} \int T\left(x_{i}\right) y_{i}^{\prime} d v \leq C\left\|\sum_{i=1}^{n} S\left(h x_{i}\right) y_{i}^{\prime}\right\|_{Y_{2} \pi Y_{1}^{\prime}}, \quad n \in \mathbb{N},
$$

for all $x_{1}, \ldots, x_{n} \in X_{1}(\mu)$ and $y_{1}^{\prime}, \ldots, y_{n}^{\prime} \in Y_{1}(v)^{\prime}$. 
Proof. First note that $\left(Y_{2} \pi Y_{1}^{\prime}\right)_{a}$ is saturated. Indeed, by taking $0<y_{2} \in Y_{2}(\nu)$ and $0<y_{1}^{\prime} \in Y_{1}(v)^{\prime}$ we have that $0<y_{2} y_{1}^{\prime} \in\left(Y_{2} \pi Y_{1}^{\prime}\right)_{a}$. Then

$$
\left(Y_{2} \pi Y_{1}^{\prime}\right)_{a}^{\prime}=\left(Y_{2} \pi Y_{1}^{\prime}\right)^{\prime}=Y_{2}^{Y_{1}^{\prime \prime}} .
$$

(b) $\Rightarrow$ (a) By Theorem 3.1 there exists $\xi^{*} \in\left(Y_{2} \pi Y_{1}^{\prime}\right)^{*}$ such that

$$
\left\langle\eta(T(x)), y^{\prime}\right\rangle=\left\langle R_{\xi *}(S(h x)), y^{\prime}\right\rangle=\left\langle\xi^{*}, S(h x) y^{\prime}\right\rangle
$$

for all $x \in X_{1}(\mu)$ and $y^{\prime} \in Y_{1}(v)^{\prime}$. Denote by $\underset{\tilde{\xi}^{*}}{\tilde{\xi}^{*}}$ the restriction of $\xi^{*}$ to $\left(Y_{2} \pi Y_{1}^{\prime}\right)_{a}$. Since $\left(Y_{2} \pi Y_{1}^{\prime}\right)_{a}$ is $\sigma$-order continuous and $\widetilde{\xi}^{*} \in\left(Y_{2} \pi Y_{1}^{\prime}\right)_{a}^{*}$, we can identify $\widetilde{\xi}^{*}$ with a function $g \in\left(Y_{2} \pi Y_{1}^{\prime}\right)_{a}^{\prime}=Y_{2}^{Y_{1}^{\prime \prime}}$, that is, $\left\langle\tilde{\xi}^{*}, z\right\rangle=\int g z d \nu$ for all $z \in$ $\left(Y_{2} \pi Y_{1}^{\prime}\right)_{a}$. Then, for all $x \in X_{1}(\mu)$ and $y^{\prime} \in Y_{1}(v)^{\prime}$, we have that

$$
\begin{aligned}
\left\langle\eta(T(x)), y^{\prime}\right\rangle & =\left\langle\xi^{*}, S(h x) y^{\prime}\right\rangle=\left\langle\widetilde{\xi}^{*}, S(h x) y^{\prime}\right\rangle \\
& =\int g S(h x) y^{\prime} d v=\left\langle\eta(g S(h x)), y^{\prime}\right\rangle,
\end{aligned}
$$

and so $T(x)=g S(h x)$.

(a) $\Rightarrow$ (b) Let $g \in Y_{2}^{Y_{1}^{\prime \prime}}=\left(Y_{2} \pi Y_{1}^{\prime}\right)^{\prime}$ be such that $T(x)=g S(h x)$ for all $x \in$ $X_{1}(\mu)$. Consider the continuous linear injection $\tilde{\eta}:\left(Y_{2} \pi Y_{1}^{\prime}\right)^{\prime} \rightarrow\left(Y_{2} \pi Y_{1}^{\prime}\right)^{*}$. Then $\tilde{\eta}(g) \in\left(Y_{2} \pi Y_{1}^{\prime}\right)^{*}$ satisfies

$$
\begin{aligned}
\left\langle R_{\widetilde{\eta}(g)}(S(h x)), y^{\prime}\right\rangle & =\left\langle\widetilde{\eta}(g), S(h x) y^{\prime}\right\rangle=\int g S(h x) y^{\prime} d \mu \\
& =\int T(x) y^{\prime} d \mu=\left\langle\eta(T(x)), y^{\prime}\right\rangle
\end{aligned}
$$

for all $x \in X_{1}(\mu)$ and $y^{\prime} \in Y_{1}(v)^{\prime}$, and so Theorem 3.1(b) holds for $\xi^{*}=\tilde{\eta}(g)$.

REMARK 3.3. We notice that the condition $y_{2} y_{1}^{\prime} \in\left(Y_{2} \pi Y_{1}^{\prime}\right)_{a}$ for all $y_{2} \in Y_{2}(v)$ and $y_{1}^{\prime} \in Y_{1}(\nu)^{\prime}$ holds when $Y_{2} \pi Y_{1}^{\prime}$ is $\sigma$-order continuous. This condition is also obtained, for instance, if any of $Y_{2}(\nu)$ or $Y_{1}(\nu)^{\prime}$ is $\sigma$-order continuous. Indeed, suppose that $Y_{2}(v)$ is $\sigma$-order continuous and take $y_{2} \in Y_{2}(v)=\left(Y_{2}\right)_{a}(v)$ and $y_{1}^{\prime} \in Y_{1}(\nu)^{\prime}$. For every $\left(A_{n}\right) \subset \Sigma$ such that $A_{n} \downarrow$ with $v\left(\cap A_{n}\right)=0$, we have that

$$
\left\|y_{2} y_{1}^{\prime} \chi_{A_{n}}\right\|_{Y_{2} \pi Y_{1}^{\prime}} \leq\left\|y_{2} \chi_{A_{n}}\right\|_{Y_{2}} \cdot\left\|y_{1}^{\prime}\right\|_{Y_{1}^{\prime}} \rightarrow 0
$$

and so $y_{2} y_{1}^{\prime} \in\left(Y_{2} \pi Y_{1}^{\prime}\right)_{a}$. We get the case where $Y_{1}(\nu)^{\prime}$ is $\sigma$-order continuous in a similar way.

This shows sufficient conditions for the product to be ( $\sigma$-)order continuous. In [10, Cor. 1] a full characterization of when a related construction-the space $Y_{1} \odot$ $Y_{2}$, defined as a pointwise product of couples of functions-is order continuous is given.

REMARK 3.4. Note that if the $\sigma$-order continuous part $X_{a}(\mu)$ of a saturated Banach function space $X(\mu)$ is also saturated, then $\|x\|_{X}=\|x\|_{X^{\prime \prime}}$ for all $x \in X_{a}(\mu)$. Indeed, for every $x \in X_{a}(\mu)$, we have that $\|x\|_{X}=\|x\|_{X_{a}^{\prime \prime}}$ since $X_{a}(\mu)$ is order 
semicontinuous and $\|x\|_{X_{a}^{\prime \prime}}=\|x\|_{X^{\prime \prime}}$ since $X_{a}(\mu)^{\prime}=X(\mu)^{\prime}$ with equal norms. Then, the norm in Corollary 3.2(b) can be computed as

$$
\begin{aligned}
\left\|\sum_{i=1}^{n} S\left(h x_{i}\right) y_{i}^{\prime}\right\|_{Y_{2} \pi Y_{1}^{\prime}} & =\left\|\sum_{i=1}^{n} S\left(h x_{i}\right) y_{i}^{\prime}\right\|_{\left(Y_{2} \pi Y_{1}^{\prime}\right)^{\prime \prime}}=\left\|\sum_{i=1}^{n} S\left(h x_{i}\right) y_{i}^{\prime}\right\|_{\left(Y_{2}^{Y_{1}^{\prime \prime}}\right)^{\prime}} \\
& =\sup _{f \in B_{Y_{2} Y_{1}^{\prime \prime}}} \int\left|f \sum_{i=1}^{n} S\left(h x_{i}\right) y_{i}^{\prime}\right| d \nu .
\end{aligned}
$$

\section{Strong Factorization Involving Schauder Basis}

Let $(\Omega, \Sigma, \mu),(\Delta, \Gamma, \nu)$ be $\sigma$-finite measure spaces, let $X_{1}(\mu), X_{2}(\mu), Y_{1}(\nu)$, $Y_{2}(\nu)$ be saturated Banach function spaces, and let $T: X_{1}(\mu) \rightarrow Y_{1}(\nu)$ and $S: X_{2}(\mu) \rightarrow Y_{2}(\nu)$ be nontrivial continuous linear operators. In this section, we assume the existence of a Schauder basis $\left(\gamma_{n}\right)$ for $Y_{1}(\nu)^{\prime}$ and denote by $\left(\gamma_{n}^{*}\right)$ the sequence of coefficient functionals with respect to this basis.

THEOREM 4.1. Assume that $Y_{2}^{Y_{1}^{\prime \prime}}$ is saturated and that any of $Y_{2}(v)$ or $Y_{1}(v)^{\prime}$ is $\sigma$-order continuous. Given $h \in X_{1}^{X_{2}}$, the following statements are equivalent:

(a) The operator $T$ factors strongly through $S$ and $M_{h}$.

(b) There exists a constant $C>0$ such that

$$
\sum_{i=1}^{n} \int T\left(x_{i}\right) \gamma_{i} d \nu \leq C\left\|\sum_{i=1}^{n} S\left(h x_{i}\right) \gamma_{i}\right\|_{Y_{2} \pi Y_{1}^{\prime}}, \quad n \in \mathbb{N},
$$

for all $x_{1}, \ldots, x_{n} \in X_{1}(\mu)$.

Moreover, if $Y_{2}(v) \subset Y_{1}(v)^{\prime \prime}$ and the functions $\left(\gamma_{n}\right)$ have pairwise disjoint supports, then conditions (a)-(b) follow from the following condition:

(c) There exists a constant $C>0$ such that

$$
\int T(x) \gamma_{n} d \nu \leq C \int\left|S(h x) \gamma_{n}\right| d \nu, \quad n \in \mathbb{N},
$$

for every $x \in X_{1}(\mu)$.

In the case where $Y_{2}^{Y_{1}^{\prime \prime}}=L^{\infty}(v)$, we have that (c) is equivalent to (a)-(b).

Proof. (a) $\Leftrightarrow$ (b) By Remark 3.3 we only have to prove that condition (b) of the present theorem implies condition (b) of Corollary 3.2. The converse implication follows by taking $y_{i}^{\prime}=\gamma_{i}$. Let $x_{1}, \ldots, x_{n} \in X_{1}(\mu)$ and $y_{1}^{\prime}, \ldots, y_{n}^{\prime} \in Y_{1}(\nu)^{\prime}$. Fix $m \in \mathbb{N}$ and denote $\left(y_{i}^{\prime}\right)^{m}=\sum_{k=1}^{m}\left\langle\gamma_{k}^{*}, y_{i}^{\prime}\right\rangle \gamma_{k}$. It follows that

$$
\begin{aligned}
\sum_{i=1}^{n} \int T\left(x_{i}\right)\left(y_{i}^{\prime}\right)^{m} d v & =\sum_{i=1}^{n} \sum_{k=1}^{m}\left\langle\gamma_{k}^{*}, y_{i}^{\prime}\right\rangle \int T\left(x_{i}\right) \gamma_{k} d v \\
& =\sum_{k=1}^{m} \int\left(\sum_{i=1}^{n}\left\langle\gamma_{k}^{*}, y_{i}^{\prime}\right\rangle T\left(x_{i}\right)\right) \gamma_{k} d v
\end{aligned}
$$




$$
\begin{aligned}
& =\sum_{k=1}^{m} \int T\left(\sum_{i=1}^{n}\left\langle\gamma_{k}^{*}, y_{i}^{\prime}\right\rangle x_{i}\right) \gamma_{k} d v \\
& \leq C\left\|\sum_{k=1}^{m} S\left(h \sum_{i=1}^{n}\left\langle\gamma_{k}^{*}, y_{i}^{\prime}\right\rangle x_{i}\right) \gamma_{k}\right\|_{Y_{2} \pi Y_{1}^{\prime}} \\
& =C\left\|\sum_{k=1}^{m} \sum_{i=1}^{n}\left\langle\gamma_{k}^{*}, y_{i}^{\prime}\right\rangle S\left(h x_{i}\right) \gamma_{k}\right\|_{Y_{2} \pi Y_{1}^{\prime}} \\
& =C\left\|\sum_{i=1}^{n} S\left(h x_{i}\right)\left(y_{i}^{\prime}\right)^{m}\right\|_{Y_{2} \pi Y_{1}^{\prime}} .
\end{aligned}
$$

Since $\left(y_{i}^{\prime}\right)^{m} \rightarrow y_{i}^{\prime}$ in $Y_{1}(v)^{\prime}$ as $m \rightarrow \infty$ and

$$
\left|\int z y_{i}^{\prime} d \nu-\int z\left(y_{i}^{\prime}\right)^{m} d \nu\right|=\left|\int z\left(y_{i}^{\prime}-\left(y_{i}^{\prime}\right)^{m}\right) d \nu\right| \leq\|z\|_{Y_{1}}\left\|y_{i}^{\prime}-\left(y_{i}^{\prime}\right)^{m}\right\|_{Y_{1}^{\prime}}
$$

for every $z \in Y_{1}(v)$, we have that $\sum_{i=1}^{n} \int T\left(x_{i}\right)\left(y_{i}^{\prime}\right)^{m} d v \rightarrow \sum_{i=1}^{n} \int T\left(x_{i}\right) y_{i}^{\prime} d v$ as $m \rightarrow \infty$. On the other hand, since

$$
\left\|z y_{i}^{\prime}-z\left(y_{i}^{\prime}\right)^{m}\right\|_{Y_{2} \pi Y_{1}^{\prime}}=\left\|z\left(y_{i}^{\prime}-\left(y_{i}^{\prime}\right)^{m}\right)\right\|_{Y_{2} \pi Y_{1}^{\prime}} \leq\|z\|_{Y_{2}}\left\|y_{i}^{\prime}-\left(y_{i}^{\prime}\right)^{m}\right\|_{Y_{1}^{\prime}}
$$

for every $z \in Y_{2}(\nu)$, we have that $\sum_{i=1}^{n} S\left(h x_{i}\right)\left(y_{i}^{\prime}\right)^{m} \rightarrow \sum_{i=1}^{n} S\left(h x_{i}\right) y_{i}^{\prime}$ in $Y_{2} \pi Y_{1}^{\prime}$ as $m \rightarrow \infty$. Then, taking the limit as $m \rightarrow \infty$ in (4.1), we obtain

$$
\sum_{i=1}^{n} \int T\left(x_{i}\right) y_{i}^{\prime} d v \leq C\left\|\sum_{i=1}^{n} S\left(h x_{i}\right) y_{i}^{\prime}\right\|_{Y_{2} \pi Y_{1}^{\prime}} .
$$

Assume that $Y_{2}(v) \subset Y_{1}(v)^{\prime \prime}$ and that the functions $\left(\gamma_{n}\right)$ have pairwise disjoint supports. Let us see that (c) implies (b). The condition $Y_{2}(v) \subset Y_{1}(v)^{\prime \prime}$ is equivalent to $L^{\infty}(v) \subset Y_{2}^{Y_{1}^{\prime \prime}}=\left(Y_{2} \pi Y_{1}^{\prime}\right)^{\prime}$, and so $Y_{2} \pi Y_{1}^{\prime} \subset\left(Y_{2} \pi Y_{1}^{\prime}\right)^{\prime \prime} \subset L^{\infty}(v)^{\prime}=L^{1}(v)$. Denote by $K$ the continuity constant of the inclusion $Y_{2} \pi Y_{1}^{\prime} \subset L^{1}(v)$. For all $n \in \mathbb{N}$ and $x_{1}, \ldots, x_{n} \in X_{1}(\mu)$, noting that $\sum_{i=1}^{n}\left|S\left(h x_{i}\right) \gamma_{i}\right|=\left|\sum_{i=1}^{n} S\left(h x_{i}\right) \gamma_{i}\right|$ pointwise (as $\left(\gamma_{k}\right)$ have disjoint supports), we have that

$$
\begin{aligned}
\sum_{i=1}^{n} \int T\left(x_{i}\right) \gamma_{i} d v & \leq C \sum_{i=1}^{n} \int\left|S\left(h x_{i}\right) \gamma_{i}\right| d v=C \int\left|\sum_{i=1}^{n} S\left(h x_{i}\right) \gamma_{i}\right| d v \\
& \leq C K\left\|\sum_{i=1}^{n} S\left(h x_{i}\right) \gamma_{i}\right\|_{Y_{2} \pi Y_{1}^{\prime}}
\end{aligned}
$$

If, moreover, $L^{\infty}(v)=Y_{2}^{Y_{1}^{\prime \prime}}$, then (a) implies (c), as if $g \in Y_{2}^{Y_{1}^{\prime \prime}}$ is such that $T(x)=$ $g S(h x)$ for all $x \in X_{1}(\mu)$, then it follows that

$$
\int T(x) \gamma_{n} d v=\int g S(h x) \gamma_{n} d v \leq \int\left|g S(h x) \gamma_{n}\right| d v \leq\|g\|_{\infty} \int\left|S(h x) \gamma_{n}\right| d v .
$$


REMARK 4.2. Note that the condition $Y_{2}(v) \subset Y_{1}(v)^{\prime \prime}$ appearing in the statement of Theorem 4.1-and also in Theorem 4.3-follows immediately from the assumption that $Y_{2}^{Y_{1}^{\prime \prime}}$ is saturated if we consider symmetric Banach function spaces on $I=[0,1]$. Indeed, in this case, since $Y_{2}^{Y_{1}^{\prime \prime}}$ is saturated, we have in particular that $Y_{2}^{Y_{1}^{\prime \prime}} \neq\{0\}$, which is equivalent to $Y_{2}(v) \subset Y_{1}(v)^{\prime \prime}$ by [9, Prop. 2.3].

Now suppose that there is also a Schauder basis $\left(\beta_{n}\right)$ for $X_{1}(\mu)$ and denote by $\left(\beta_{n}^{*}\right)$ the sequence of its coefficient functionals. Then, the equivalent inequalities for the strong factorization can be relaxed.

THEOREM 4.3. Assume that $Y_{2}^{Y_{1}^{\prime \prime}}$ is saturated and that any of $Y_{2}(v)$ or $Y_{1}(v)^{\prime}$ is $\sigma$-order continuous. Given $h \in X_{1}^{X_{2}}$, the following statements are equivalent:

(a) The operator $T$ factors strongly through $S$ and $M_{h}$.

(b) There exists $g \in Y_{2}^{Y_{1}^{\prime \prime}}$ such that $T\left(\beta_{n}\right)=g S\left(h \beta_{n}\right)$ for each $n \in \mathbb{N}$.

(c) There exists a constant $C>0$ such that

$$
\sum_{i=1}^{n} \sum_{j=1}^{m} r_{i j} \int T\left(\beta_{j}\right) \gamma_{i} d \nu \leq C\left\|\sum_{i=1}^{n} \sum_{j=1}^{m} r_{i j} S\left(h \beta_{j}\right) \gamma_{i}\right\|_{Y_{2} \pi Y_{1}^{\prime}}, \quad n, m \in \mathbb{N},
$$

for every $\left(r_{i j}\right) \subset B_{\ell}$.

Moreover, if $Y_{2}(v) \subset Y_{1}(v)^{\prime \prime}$ and the functions $\left(\gamma_{n}\right)$ have pairwise disjoint support, then conditions (a)-(c) follow from the following condition:

(d) There exists a constant $C>0$ such that the inequality

$$
\sum_{j=1}^{m} r_{j} \int T\left(\beta_{j}\right) \gamma_{n} d v \leq C \int\left|\sum_{j=1}^{m} r_{j} S\left(h \beta_{j}\right) \gamma_{n}\right| d v
$$

for all $n, m \in \mathbb{N}$ and $\left(r_{j}\right) \subset B_{\ell}$.

In the case where $L^{\infty}(v)=Y_{2}^{Y_{1}^{\prime \prime}}$, we have that (d) is equivalent to (a)-(c).

Proof. (a) $\Rightarrow$ (b) Let $g \in Y_{2}^{Y_{1}^{\prime \prime}}$ be such that $T(x)=g S(h x)$ for all $x \in X_{1}(\mu)$. In particular, for $x=\beta_{n}$, we obtain (b).

(b) $\Rightarrow$ (c) Since $g \in Y_{2}^{Y_{1}^{\prime \prime}}=\left(Y_{2} \pi Y_{1}^{\prime}\right)^{\prime}$ for all $n, m \in \mathbb{N}$ and $\left(r_{i j}\right) \subset B_{\ell}$, it follows that

$$
\begin{aligned}
\sum_{i=1}^{n} \sum_{j=1}^{m} r_{i j} \int T\left(\beta_{j}\right) \gamma_{i} d v & =\sum_{i=1}^{n} \sum_{j=1}^{m} r_{i j} \int g S\left(h \beta_{j}\right) \gamma_{i} d v \\
& =\int g \sum_{i=1}^{n} \sum_{j=1}^{m} r_{i j} S\left(h \beta_{j}\right) \gamma_{i} d v \\
& \leq \int\left|g \sum_{i=1}^{n} \sum_{j=1}^{m} r_{i j} S\left(h \beta_{j}\right) \gamma_{i}\right| d v
\end{aligned}
$$




$$
\leq\|g\|_{\left(Y_{2} \pi Y_{1}^{\prime}\right)^{\prime}}\left\|\sum_{i=1}^{n} \sum_{j=1}^{m} r_{i j} S\left(h \beta_{j}\right) \gamma_{i}\right\|_{Y_{2} \pi Y_{1}^{\prime}} .
$$

(c) $\Rightarrow$ (a) Let us show that the condition (b) of Theorem 4.1 holds. Let $x_{1}, \ldots, x_{n} \in X_{1}(\mu)$, which can be assumed to be nonnull. Fix $m \in \mathbb{N}$ large enough such that $\left(x_{i}\right)^{m}=\sum_{j=1}^{m}\left\langle\beta_{j}^{*}, x_{i}\right\rangle \beta_{j} \neq 0$ and denote $\alpha=\max \underset{\substack{i=1, \ldots, n \\ j=1, \ldots, m}}{ }\left|\left\langle\beta_{j}^{*}, x_{i}\right\rangle\right|$. By taking $r_{i j}=\frac{\left\langle\beta_{j}^{*}, x_{i}\right\rangle}{\alpha}$ it follows that

$$
\begin{aligned}
\sum_{i=1}^{n} \int T\left(\left(x_{i}\right)^{m}\right) \gamma_{i} d v & =\sum_{i=1}^{n} \sum_{j=1}^{m}\left\langle\beta_{j}^{*}, x_{i}\right\rangle \int T\left(\beta_{j}\right) \gamma_{i} d v \\
& =\alpha \sum_{i=1}^{n} \sum_{j=1}^{m} r_{i j} \int T\left(\beta_{j}\right) \gamma_{i} d v \\
& \leq \alpha C\left\|\sum_{i=1}^{n} \sum_{j=1}^{m} r_{i j} S\left(h \beta_{j}\right) \gamma_{i}\right\|_{Y_{2} \pi Y_{1}^{\prime}} \\
& =C\left\|\sum_{i=1}^{n} \sum_{j=1}^{m}\left\langle\beta_{j}^{*}, x_{i}\right\rangle S\left(h \beta_{j}\right) \gamma_{i}\right\|_{Y_{2} \pi Y_{1}^{\prime}} \\
& =C\left\|\sum_{i=1}^{n} S\left(h\left(x_{i}\right)^{m}\right) \gamma_{i}\right\|_{Y_{2} \pi Y_{1}^{\prime}}
\end{aligned}
$$

Denoting by $\|T\|$ the operator norm of $T$, since $\left(x_{i}\right)^{m} \rightarrow x_{i}$ in $X_{1}(\mu)$ as $m \rightarrow \infty$ and

$$
\begin{aligned}
\left|\int T\left(x_{i}\right) z d v-\int T\left(\left(x_{i}\right)^{m}\right) z d v\right| & =\left|\int T\left(x_{i}-\left(x_{i}\right)^{m}\right) z d v\right| \\
& \leq\|z\|_{Y_{1}^{\prime}}\left\|T\left(x_{i}-\left(x_{i}\right)^{m}\right)\right\|_{Y_{1}} \\
& \leq\|z\|_{Y_{1}^{\prime}}\|T\|\left\|x_{i}-\left(x_{i}\right)^{m}\right\|_{X_{1}}
\end{aligned}
$$

for every $z \in Y_{1}(v)^{\prime}$, we have that $\sum_{i=1}^{n} \int T\left(\left(x_{i}\right)^{m}\right) \gamma_{i} d v \rightarrow \sum_{i=1}^{n} \int T\left(x_{i}\right) \gamma_{i} d v$ as $m \rightarrow \infty$. On other hand, we have

$$
\begin{aligned}
\left\|S\left(h x_{i}\right) z-S\left(h\left(x_{i}\right)^{m}\right) z\right\|_{Y_{2} \pi Y_{1}^{\prime}} & =\left\|S\left(h\left(x_{i}-\left(x_{i}\right)^{m}\right)\right) z\right\|_{Y_{2} \pi Y_{1}^{\prime}} \\
& \leq\|z\|_{Y_{1}^{\prime}}\left\|S\left(h\left(x_{i}-\left(x_{i}\right)^{m}\right)\right)\right\|_{Y_{2}} \\
& \leq\|z\|_{Y_{1}^{\prime}}\|S\|\left\|h\left(x_{i}-\left(x_{i}\right)^{m}\right)\right\|_{X_{2}} \\
& \leq\|z\|_{Y_{1}^{\prime}}\|S\|\|h\|_{X_{1}^{X_{2}}}\left\|x_{i}-\left(x_{i}\right)^{m}\right\|_{X_{1}}
\end{aligned}
$$

for every $z \in Y_{1}(v)^{\prime}$, we have that $\sum_{i=1}^{n} S\left(h\left(x_{i}\right)^{m}\right) \gamma_{i} \rightarrow \sum_{i=1}^{n} S\left(h x_{i}\right) \gamma_{i}$ in $Y_{2} \pi Y_{1}^{\prime}$ as $m \rightarrow \infty$. Then, taking the limit as $m \rightarrow \infty$ in (4.2), we obtain

$$
\sum_{i=1}^{n} \int T\left(x_{i}\right) \gamma_{i} d \nu \leq C\left\|\sum_{i=1}^{n} S\left(h x_{i}\right) \gamma_{i}\right\|_{Y_{2} \pi Y_{1}^{\prime}} .
$$


Assume that $Y_{2}(v) \subset Y_{1}(v)^{\prime \prime}$ and that the functions $\left(\gamma_{n}\right)$ have pairwise disjoint supports. We have already noted that in this case, $Y_{2} \pi Y_{1}^{\prime} \subset L^{1}(v)$ (denote by $K$ its continuity constant), $\sum_{i=1}^{n}\left|f_{i} \gamma_{i}\right|=\left|\sum_{i=1}^{n} f_{i} \gamma_{i}\right|$ pointwise for every $n \in \mathbb{N}$, and $\left(f_{i}\right) \subset L^{0}(v)$. Let us show that (d) implies (c). For all $n, m \in \mathbb{N}$ and $\left(r_{i j}\right) \subset B_{\ell}$, we have that

$$
\begin{aligned}
\sum_{i=1}^{n} \sum_{j=1}^{m} r_{i j} \int T\left(\beta_{j}\right) \gamma_{i} d \nu & \leq C \sum_{i=1}^{n} \int\left|\sum_{j=1}^{m} r_{i j} S\left(h \beta_{j}\right) \gamma_{i}\right| d \nu \\
& =C \int\left|\sum_{i=1}^{n} \sum_{j=1}^{m} r_{i j} S\left(h \beta_{j}\right) \gamma_{i}\right| d \nu \\
& \leq C K\left\|\sum_{i=1}^{n} \sum_{j=1}^{m} r_{i j} S\left(h \beta_{j}\right) \gamma_{i}\right\|_{Y_{2} \pi Y_{1}^{\prime}} .
\end{aligned}
$$

If moreover $L^{\infty}(v)=Y_{2}^{Y_{1}^{\prime \prime}}$, then (a) implies (d), as if $g \in Y_{2}^{Y_{1}^{\prime \prime}}$ is such that $T(x)=$ $g S(h x)$ for all $x \in X_{1}(\mu)$, then it follows that

$$
\begin{aligned}
& \sum_{j=1}^{m} r_{j} \int T\left(\beta_{j}\right) \gamma_{n} d \nu \\
& \quad=\sum_{j=1}^{m} r_{j} \int g S\left(h \beta_{j}\right) \gamma_{n} d \nu=\int g \sum_{j=1}^{m} r_{j} S\left(h \beta_{j}\right) \gamma_{n} d \nu \\
& \quad \leq \int\left|g \sum_{j=1}^{m} r_{j} S\left(h \beta_{j}\right) \gamma_{n}\right| d \nu \leq\|g\|_{\infty} \int\left|\sum_{j=1}^{m} r_{j} S\left(h \beta_{j}\right) \gamma_{n}\right| d \nu .
\end{aligned}
$$

\section{Examples: The Fourier and Cesàro Operators}

In this section, we show how the results obtained in the previous one can be applied in concrete contexts. In particular, we will deal with the Fourier operator acting in different weighted $L^{p}$-spaces, we will show factorization through infinite matrices, and, as a particular case, we will analyze the case provided by the Cesàro operator.

\subsection{Strong Factorization Through the Fourier Operator}

Consider the measure space given by the interval $\mathbb{T}=[-\pi, \pi]$, its Borel $\sigma-$ algebra, and the Lebesgue measure $m$, and denote by $\left(\phi_{n}\right)$ the real trigonometric system on $\mathbb{T}$, that is,

$$
\phi_{n}(x)= \begin{cases}\frac{1}{\sqrt{2 \pi}} & \text { if } n=1, \\ \frac{\cos k x}{\sqrt{\pi}} & \text { if } n=2 k, \\ \frac{\sin k x}{\sqrt{\pi}} & \text { if } n=2 k+1 .\end{cases}
$$


Note that $\int_{-\pi}^{\pi} \phi_{i}(x) \phi_{j}(x) d m=0$ if $i \neq j$ and $\int_{-\pi}^{\pi} \phi_{i}(x) \phi_{i}(x) d m=1$. Each function $f \in L^{1}(m)$ is associated with its Fourier series $S(f)=\sum_{n \geq 1} a_{n} \phi_{n}$ with $a_{n}=\int_{\mathbb{T}} f \phi_{n} d m$. If $f \in L^{r}(m)$ for $1<r<\infty$, then $S(f)$ converges to $f$ in $L^{r}(m)$, and so $\left(\phi_{n}\right)$ is a Schauder basis on $L^{r}(m)$.

Let $\mathcal{F}$ be the Fourier operator defined by

$$
\mathcal{F}(f)=\left(\int_{\mathbb{T}} f \phi_{n} d m\right), \quad f \in L^{1}(m) .
$$

The Hausdorff-Young inequality (see, e.g., [7, (8.5.7)]) guarantees that

$$
\mathcal{F}: L^{r}(m) \rightarrow \ell^{r^{\prime}}
$$

is a well-defined continuous operator for every $1<r \leq 2$.

Fix $1<r \leq 2, r \leq p<\infty, 1<q \leq \infty$, and let $T: L^{p}(m) \rightarrow \ell^{q}$ be a nontrivial continuous linear operator. We have that $\left(\phi_{n}\right)$ is a Schauder basis for $L^{p}(m)$ (as $1<p<\infty)$ and $\left(e^{n}\right)$ is a Schauder basis for $\left(\ell^{q}\right)^{\prime}$ (as $\left.q>1\right)$. Also, $L^{p}(m) \subset$ $L^{r}(m)($ as $r \leq p)$, and so $\chi_{\mathbb{T}} \in\left(L^{p}\right)^{L^{r}}$.

Proposition 5.1. The following statements are equivalent:

(a) The operator $T$ factors strongly through $\mathcal{F}$, that is, there exists $g \in \ell^{s_{r^{\prime} q}}$ such that

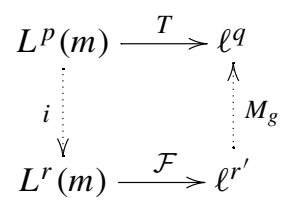

(see (2.1) in the preliminaries for the definition of $s_{r^{\prime} q}$ ).

(b) $T\left(\phi_{n}\right)_{i}=0$ for all $i \neq n$ and $\left(T\left(\phi_{i}\right)_{i}\right) \in \ell^{S_{r^{\prime} q}}$.

(c) There exists a constant $C>0$ such that

$$
\sum_{i=1}^{n} \sum_{j=1}^{m} r_{i j} T\left(\phi_{j}\right)_{i} \leq C\left(\sum_{i=1}^{\min \{n, m\}}\left|r_{i i}\right|^{s_{r^{\prime} q}^{\prime}}\right)^{\frac{1}{s_{r^{\prime} q}^{\prime}}}, \quad n, m \in \mathbb{N},
$$

for every $\left(r_{i j}\right) \subset B_{\ell}$.

Moreover, in the case where $r^{\prime} \leq q$, conditions (a)-(c) are equivalent to

(d) There exists a constant $C>0$ such that

$$
\sum_{j=1}^{m} r_{j} T\left(\phi_{j}\right)_{n} \leq C \begin{cases}\left|r_{n}\right| & \text { if } n \leq m, \\ 0 & \text { if } n>m,\end{cases}
$$

for each $n, m \in \mathbb{N}$ and all $\left(r_{j}\right) \subset B_{\ell^{\infty}}$.

Proof. Note that both $\ell^{r^{\prime}}$ and $\left(\ell^{q}\right)^{\prime}$ are $\sigma$-order continuous (as $r, q>1$ ) and that $\left(\ell^{r^{\prime}}\right)^{\left(\ell^{q}\right)^{\prime \prime}}=\left(\ell^{r^{\prime}}\right)^{\ell^{q}}=\ell^{s_{r^{\prime} q}}$, where $s_{r^{\prime} q}$ is defined as in (2.1). For the equivalence among (a), (b), and (c), let us see that conditions (b) and (c) are just respectively conditions (b) and (c) of Theorem 4.3 rewritten for $X_{1}(\mu)=L^{p}(m), X_{2}(\mu)=$ 
$L^{r}(m), Y_{1}(v)=\ell^{q}(v$ being the counting measure $\lambda$ on $\mathbb{N}), Y_{2}(v)=\ell^{r^{\prime}}, S=\mathcal{F}$, $h=\chi_{\mathbb{T}},\left(\beta_{n}\right)=\left(\phi_{n}\right)$, and $\left(\gamma_{n}\right)=\left(e^{n}\right)$.

(b) $\Rightarrow$ Theorem 4.3(b). Take $g=\left(T\left(\phi_{i}\right)_{i}\right) \in \ell^{s_{r^{\prime} q}}$. Then, for all $n, i \in \mathbb{N}$, we have that $T\left(\phi_{n}\right)_{i}=T\left(\phi_{i}\right)_{i} \mathcal{F}\left(\phi_{n}\right)_{i}=g_{i} \mathcal{F}\left(\phi_{n}\right)_{i}$, and so $T\left(\phi_{n}\right)=g \mathcal{F}\left(\phi_{n}\right)$.

Theorem $4.3(\mathrm{~b}) \Rightarrow(\mathrm{b})$. Let $g \in \ell^{S_{r^{\prime} q}}$ be such that $T\left(\phi_{n}\right)=g \mathcal{F}\left(\phi_{n}\right)$ for all $n \in \mathbb{N}$. Then

$$
T\left(\phi_{n}\right)_{i}=g_{i} \mathcal{F}\left(\phi_{n}\right)_{i}= \begin{cases}g_{i} & \text { if } i=n, \\ 0 & \text { if } i \neq n,\end{cases}
$$

and so $\left(T\left(\phi_{i}\right)_{i}\right)=g \in \ell^{S_{r^{\prime} q}}$.

(c) $\Leftrightarrow$ Theorem 4.3(c). From Remark 3.4, noting that $\left(\ell^{r^{\prime}} \pi\left(\ell^{q}\right)^{\prime}\right)^{\prime \prime}=$ $\left(\left(\ell^{r^{\prime}}\right)^{\left(\ell^{q}\right)^{\prime \prime}}\right)^{\prime}=\left(\ell^{s_{r^{\prime} q}}\right)^{\prime}=\ell^{s_{r^{\prime} q}^{\prime}}$ with equals norms and $s_{r^{\prime} q}^{\prime}<\infty\left(\right.$ as $\left.s_{r^{\prime} q}>1\right)$, for all $n, m \in \mathbb{N}$ and $\left(r_{i j}\right) \subset B_{\ell}$, it follows that

$$
\begin{aligned}
\left\|\sum_{i=1}^{n} \sum_{j=1}^{m} r_{i j} \mathcal{F}\left(\phi_{j}\right) e^{i}\right\|_{\ell^{r^{\prime} \pi(\ell q)^{\prime}}} & =\left\|\sum_{i=1}^{n} \sum_{j=1}^{m} r_{i j} \mathcal{F}\left(\phi_{j}\right) e^{i}\right\|_{\left(\ell^{\left.r^{\prime} \pi(\ell q)^{\prime}\right)^{\prime \prime}}\right.} \\
& =\left\|\sum_{i=1}^{n} \sum_{j=1}^{m} r_{i j} \mathcal{F}\left(\phi_{j}\right) e^{i}\right\|_{\ell^{s_{r^{\prime} q}}} \\
& =\left\|\sum_{i=1}^{n} \sum_{j=1}^{m} r_{i j} \mathcal{F}\left(\phi_{j}\right)_{i} e^{i}\right\|_{\ell^{s^{\prime} r^{\prime} q}} \\
& =\left(\sum_{i=1}^{n}\left|\sum_{j=1}^{m} r_{i j} \mathcal{F}\left(\phi_{j}\right)_{i}\right|^{s_{r^{\prime} q}^{\prime}}\right)^{\frac{1}{s_{r^{\prime} q}}} \\
& =\left(\sum_{i=1}^{\min \{n, m\}}\left|r_{i i}\right|^{s_{r^{\prime} q}}\right)^{\frac{1}{s_{r^{\prime} q}}}
\end{aligned}
$$

and

$$
\sum_{i=1}^{n} \sum_{j=1}^{m} r_{i j} \int T\left(\phi_{j}\right) e^{i} d \lambda=\sum_{i=1}^{n} \sum_{j=1}^{m} r_{i j} T\left(\phi_{j}\right)_{i}
$$

In the case where $r^{\prime} \leq q$, we have that $s_{r^{\prime} q}=\infty$, and so $\left(\ell^{r^{\prime}}\right)^{\left(\ell^{q}\right)^{\prime \prime}}=\ell^{\infty}$. Then (d) is equivalent to (a)-(c) as (d) is rewritten condition (d) of Theorem 4.3. Indeed,

$$
\sum_{j=1}^{m} r_{j} \int T\left(\phi_{j}\right) e^{n} d \lambda=\sum_{j=1}^{m} r_{j} T\left(\phi_{j}\right)_{n}
$$

and

$$
\int\left|\sum_{j=1}^{m} r_{j} \mathcal{F}\left(\phi_{j}\right) e^{n}\right| d \lambda=\left|\sum_{j=1}^{m} r_{j} \mathcal{F}\left(\phi_{j}\right)_{n}\right|= \begin{cases}\left|r_{n}\right| & \text { if } n \leq m, \\ 0 & \text { if } n>m .\end{cases}
$$




\subsection{Strong Factorization for Infinite Matrices and the Cesàro Operator}

Consider the measure space $(\mathbb{N}, \mathcal{P}(\mathbb{N}), \lambda)$ with $\lambda$ being the counting measure on $\mathbb{N}$. Let $X_{1}(\lambda), X_{2}(\lambda), Y_{1}(\lambda), Y_{2}(\lambda)$ be saturated Banach function spaces in which $\left(e^{n}\right)$ is a Schauder basis and $T: X_{1}(\lambda) \rightarrow Y_{1}(\lambda)$, and let $S: X_{2}(\lambda) \rightarrow Y_{2}(\lambda)$ be nontrivial continuous linear operators. Then the operators $T$ and $S$ can be described by infinite matrices $\left(a_{i j}\right)$ and $\left(b_{i j}\right)$, respectively, namely $a_{i j}=T\left(e^{j}\right)_{i}$ and $b_{i j}=S\left(e^{j}\right)_{i}$. We also require that $\left(e^{n}\right)$ is a Schauder basis for $Y_{1}(\lambda)^{\prime}$.

Proposition 5.2. Assume that $Y_{2}^{Y_{1}^{\prime \prime}}$ is saturated and that any of $Y_{2}(\lambda)$ or $Y_{1}(\lambda)^{\prime}$ is $\sigma$-order continuous. Given $h \in X_{1}^{X_{2}}$, the following statements are equivalent:

(a) The operator $T$ factors strongly through $S$ and $M_{h}$.

(b) There exists $g \in Y_{2}^{Y_{1}^{\prime \prime}}$ such that $\frac{a_{i j}}{b_{i j}}=g_{i} h_{j}$ whenever $b_{i j} \neq 0$ and $a_{i j}=0$ whenever $b_{i j}=0$.

(c) There exists a constant $C>0$ such that

$$
\sum_{i=1}^{n} \sum_{j=1}^{m} r_{i j} a_{i j} \leq C\left\|\sum_{i=1}^{n}\left(\sum_{j=1}^{m} h_{j} r_{i j} b_{i j}\right) e^{i}\right\|_{Y_{2} \pi Y_{1}^{\prime}}, \quad n, m \in \mathbb{N},
$$

for every $\left(r_{i j}\right) \subset B_{\ell}$.

Moreover, if $Y_{2}(\lambda) \subset Y_{1}(\lambda)^{\prime \prime}$, then conditions (a)-(c) follow from the following condition:

(d) There exists a constant $C>0$ such that the inequality

$$
\sum_{j=1}^{m} r_{j} a_{n j} \leq C\left|\sum_{j=1}^{m} h_{j} r_{j} b_{n j}\right|, \quad n, m \in \mathbb{N},
$$

for every $\left(r_{j}\right) \subset B_{\ell}$.

In the case where $\ell^{\infty}=Y_{2}^{Y_{1}^{\prime \prime}}$, we have that $(\mathrm{d})$ is equivalent to $(\mathrm{a})-(\mathrm{c})$.

Proof. We only have to see that conditions (b), (c), and (d) are just respectively conditions (b), (c), and (d) of Theorem 4.3 rewritten for $\mu=v$ being the counting measure $\lambda$ and $\left(\beta_{n}\right)=\left(\gamma_{n}\right)=\left(e^{n}\right)$. Note that for every $i, j \in \mathbb{N}$, we have that $a_{i j}=T\left(e^{j}\right)_{i}$ and $g_{i} h_{j} b_{i j}=g_{i} h_{j} S\left(e^{j}\right)_{i}=g_{i} S\left(h_{j} e^{j}\right)_{i}=g_{i} S\left(h e^{j}\right)_{i}$. So (b) $\Leftrightarrow$ Theorem 4.3(b). Since $\int T\left(e^{j}\right) e^{i} d \lambda=T\left(e^{j}\right)_{i}=a_{i j}$ and $S\left(h e^{j}\right) e^{i}=S\left(h_{j} e^{j}\right) e^{i}=$ $h_{j} S\left(e^{j}\right) e^{i}=h_{j} S\left(e^{j}\right)_{i} e^{i}=h_{j} b_{i j} e^{i}$, we have that (c) $\Leftrightarrow$ Theorem 4.3(c). Moreover, as

$$
\int\left|\sum_{j=1}^{m} r_{j} S\left(h e^{j}\right) e^{n}\right| d \lambda=\int\left|\sum_{j=1}^{m} r_{j} h_{j} b_{n j} e^{n}\right| d \lambda=\left|\sum_{j=1}^{m} r_{j} h_{j} b_{n j}\right|,
$$

it follows that $(\mathrm{d}) \Leftrightarrow$ Theorem $4.3(\mathrm{~d})$.

Let $\mathcal{C}$ be the Cesàro operator that maps a real sequence $x=\left(x_{n}\right)$ into the sequence of its Cesàro means $\mathcal{C}(x)=\left(\frac{1}{n} \sum_{i=1}^{n} x_{i}\right)$. It is well known that $\mathcal{C}: \ell^{r} \rightarrow \ell^{r}$ continuously for every $1<r<\infty$ (see [7, Thm. 326]), and it can be described by the 
infinite matrix $\left(b_{i j}\right)$ where $b_{i j}=\frac{1}{i}$ if $j \leq i$ and $b_{i j}=0$ if $j>i$, that is,

$$
\left(b_{i j}\right)=\left(\begin{array}{cccccc}
1 & 0 & 0 & 0 & 0 & \ldots \\
\frac{1}{2} & \frac{1}{2} & 0 & 0 & 0 & \ldots \\
\frac{1}{3} & \frac{1}{3} & \frac{1}{3} & 0 & 0 & \ldots \\
\frac{1}{4} & \frac{1}{4} & \frac{1}{4} & \frac{1}{4} & 0 & \ldots \\
\vdots & \vdots & \vdots & \vdots & \vdots & \ddots
\end{array}\right) .
$$

Fix $1 \leq p<\infty, 1<q, r<\infty$ and let $T: \ell^{p} \rightarrow \ell^{q}$ be the nontrivial continuous operator described by the infinite matrix $\left(a_{i j}\right)$ with $a_{i j}=T\left(e^{j}\right)_{i}$. Note that $\left(e^{n}\right)$ is a Schauder basis on $\ell^{p}, \ell^{q}, \ell^{r}$, and $\left(\ell^{q}\right)^{\prime}$.

Proposition 5.3. Let $h \in \ell^{s_{p r}}$ (see (2.1) for the definition of $s_{p r}$ ). The following statements are equivalent:

(a) The operator $T$ factors strongly through $\mathcal{C}$ and $M_{h}$, that is, there exists $g \in$ $\ell^{s_{r q}}$ such that

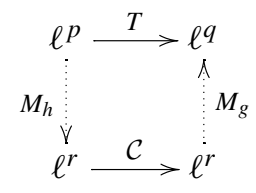

(b) There exists $g \in \ell^{s_{r q}}$ such that

$$
a_{i j}= \begin{cases}\frac{g_{i} h_{j}}{i} & \text { if } j \leq i \\ 0 & \text { if } j>i .\end{cases}
$$

(c) There exists a constant $C>0$ such that

$$
\sum_{i=1}^{n} \sum_{j=1}^{m} r_{i j} a_{i j} \leq C\left(\left.\left.\sum_{i=1}^{n} \frac{1}{i_{r q}^{\prime}}\right|^{\min \{i, m\}} \sum_{j=1}^{s_{j}} r_{i j}\right|^{s_{r q}^{\prime}}\right)^{\frac{1}{s_{r q}^{\prime}}}, \quad n, m \in \mathbb{N},
$$

for every $\left(r_{i j}\right) \in B_{\ell}$.

Moreover, in the case where $r \leq q$, conditions (a)-(c) are equivalent to the following condition:

(d) There exists a constant $C>0$ such that

$$
\sum_{j=1}^{m} r_{j} a_{n j} \leq C \frac{1}{n}\left|\sum_{j=1}^{\min \{n, m\}} h_{j} r_{j}\right|, \quad n, m \in \mathbb{N},
$$

for every $\left(r_{j}\right) \subset B_{\ell}$.

Proof. Note that both $\ell^{r}$ and $\left(\ell^{q}\right)^{\prime}$ are $\sigma$-order continuous (as $r<\infty$ and $q>1$ ), $\left(\ell^{r}\right)^{\left(\ell^{q}\right)^{\prime \prime}}=\left(\ell^{r}\right)^{\ell^{q}}=\ell^{s_{r q}}$, and $\left(\ell^{p}\right)^{\ell^{r}}=\ell^{s_{p r}}$. Also note that if $r \leq q$, then $s_{r q}=\infty$, and so $\left(\ell^{r}\right)^{\left(\ell^{q}\right)^{\prime \prime}}=\ell^{\infty}$. Then we only have to see that $(\mathrm{b}),(\mathrm{c}),(\mathrm{d})$ are just rewritten, respectively, conditions (b), (c), (d) of Proposition 5.2 for $X_{1}(\lambda)=\ell^{p}, X_{2}(\lambda)=$ $\ell^{r}, Y_{1}(\lambda)=\ell^{q}, Y_{2}(\lambda)=\ell^{r}$, and $S=\mathcal{C}$. 
As noted before, the elements of the matrix of $\mathcal{C}$ are $b_{i j}=\frac{1}{i}$ if $j \leq i$ and $b_{i j}=0$ if $j>i$, so (b) $\Leftrightarrow$ Proposition 5.2(b).

By Remark 3.4 and noting that $\left(\ell^{r} \pi\left(\ell^{q}\right)^{\prime}\right)^{\prime \prime}=\left(\left(\ell^{r}\right)^{\left(\ell^{q}\right)^{\prime \prime}}\right)^{\prime}=\left(\ell^{s_{r q}}\right)^{\prime}=\ell^{s_{r q}^{\prime}}$ with equals norms and $s_{r q}^{\prime}<\infty$ (as $s_{r q}>1$ ), for all $n, m \in \mathbb{N}$ and $\left(r_{j}\right) \subset B_{\ell}$, it follows that

$$
\begin{aligned}
\left\|\sum_{i=1}^{n}\left(\sum_{j=1}^{m} h_{j} r_{i j} b_{i j}\right) e^{i}\right\|_{\ell^{r} \pi(\ell q)^{\prime}} & =\left\|\sum_{i=1}^{n}\left(\sum_{j=1}^{m} h_{j} r_{i j} b_{i j}\right) e^{i}\right\|_{\left(\ell^{r} \pi(\ell q)^{\prime}\right)^{\prime \prime}} \\
& =\left\|\sum_{i=1}^{n}\left(\sum_{j=1}^{m} h_{j} r_{i j} b_{i j}\right) e^{i}\right\|_{\ell^{s_{r q}^{\prime}}} \\
& =\left(\sum_{i=1}^{n}\left|\sum_{j=1}^{m} h_{j} r_{i j} b_{i j}\right|^{s_{r q}^{\prime}}\right)^{\frac{1}{s_{r q}^{\prime}}} \\
& =\left(\left.\left.\sum_{i=1}^{n} \frac{1}{i^{s_{r q}^{\prime}}}\right|_{j=1} ^{\min \{i, m\}} h_{j} r_{i j}\right|^{s_{r q}^{\prime}}\right)^{\frac{1}{s_{r q}^{\prime}}} .
\end{aligned}
$$

Hence (c) $\Leftrightarrow$ Proposition 5.2(c).

(d) $\Leftrightarrow$ Proposition 5.2(d) holds as

$$
\sum_{j=1}^{m} h_{j} r_{j} b_{n j}=\frac{1}{n} \sum_{j=1}^{\min \{n, m\}} h_{j} r_{j} .
$$

We next present characterizations of matrices that factor strongly through the Cesàro operator.

Proposition 5.4. Let $h \in \ell^{s_{p r}}$ and suppose that $h_{1} \neq 0$. The following statements are equivalent:

(a) The operator $T$ factors strongly through $\mathcal{C}$ and $M_{h}$.

(b) $a_{i j}=0$ for $j>i, a_{i j}=\frac{h_{j} a_{i 1}}{h_{1}}$ for $j \leq i$, and $\left(i a_{i 1}\right) \in \ell^{s_{r q}}$.

(c) The matrix of $T$ looks as

$$
\left(\begin{array}{cccccc}
h_{1} \alpha_{1} & 0 & 0 & 0 & 0 & \ldots \\
h_{1} \alpha_{2} & h_{2} \alpha_{2} & 0 & 0 & 0 & \ldots \\
h_{1} \alpha_{3} & h_{2} \alpha_{3} & h_{3} \alpha_{3} & 0 & 0 & \ldots \\
h_{1} \alpha_{4} & h_{2} \alpha_{4} & h_{3} \alpha_{4} & h_{4} \alpha_{4} & 0 & \ldots \\
h_{1} \alpha_{5} & h_{2} \alpha_{5} & h_{3} \alpha_{5} & h_{4} \alpha_{5} & h_{5} \alpha_{5} & \ldots \\
\vdots & \vdots & \vdots & \vdots & \vdots & \ddots
\end{array}\right),
$$

where $\left(\alpha_{n}\right) \in \mathbb{R}^{\mathbb{N}}$ is such that $\left(n \alpha_{n}\right) \in \ell^{s_{r q}}$.

Proof. (a) $\Rightarrow$ (b) By Proposition 5.3 there exists $g \in \ell^{s_{r q}}$ such that

$$
a_{i j}= \begin{cases}\frac{g_{i} h_{j}}{i} & \text { if } j \leq i, \\ 0 & \text { if } j>i .\end{cases}
$$


Then $a_{i 1}=\frac{g_{i} h_{1}}{i}$ for all $i$ and so $a_{i j}=\frac{h_{j} a_{i 1}}{h_{1}}$ for every $j \leq i$. Also note that $\left(i a_{i 1}\right)_{i}=h_{1} g \in \ell^{s_{r q}}$.

(b) $\Rightarrow$ (c) Taking $\left(\alpha_{n}\right)=\left(\frac{a_{n 1}}{h_{1}}\right)$, we have that $h_{j} \alpha_{i}=\frac{h_{j} a_{i 1}}{h_{1}}=a_{i j}$ for all $j \leq i$ and $\left(n \alpha_{n}\right)=\frac{1}{h_{1}}\left(n a_{n 1}\right) \in \ell^{s_{r q}}$.

(c) $\Rightarrow$ (a) Taking $g=\left(i \alpha_{i}\right) \in \ell^{s_{r q}}$, it follows that

$$
a_{i j}= \begin{cases}h_{j} \alpha_{i}=\frac{g_{i} h_{j}}{i} & \text { if } j \leq i, \\ 0 & \text { if } j>i .\end{cases}
$$

Then, by Proposition 5.3, (a) holds.

If $T$ factors strongly through $\mathcal{C}$ and $M_{h}$, then there exists $h_{j} \neq 0$ as $T$ is nontrivial. So, given $0 \neq h \in \ell^{s_{p r}}$ and denoting $j_{0}=\min \left\{j \in \mathbb{N}: h_{j} \neq 0\right\}$, similarly to Proposition 5.4, we have that $T$ factors strongly through $\mathcal{C}$ and $M_{h}$ if and only if its matrix looks as

$$
\left(\begin{array}{cccccccc}
0 & \cdots & 0 & 0 & 0 & 0 & 0 & \cdots \\
\vdots & & \vdots & \vdots & \vdots & \vdots & \vdots & \\
0 & \cdots & 0 & 0 & 0 & 0 & 0 & \cdots \\
0 & \cdots & 0 & h_{j_{0}} \alpha_{1} & 0 & 0 & 0 & \cdots \\
0 & \cdots & 0 & h_{j 0} \alpha_{2} & h_{j 0+1} \alpha_{2} & 0 & 0 & \cdots \\
0 & \cdots & 0 & h_{j 0} \alpha_{3} & h_{j_{0}+1} \alpha_{3} & h_{j_{0}+2} \alpha_{3} & 0 & \cdots \\
0 & \cdots & 0 & h_{j 0} \alpha_{4} & h_{j 0+1} \alpha_{4} & h_{j_{0}+2} \alpha_{4} & h_{j_{0}+3} \alpha_{4} & \cdots \\
\vdots & & \vdots & \vdots & \vdots & \vdots & \vdots & \ddots
\end{array}\right)
$$

for some $\left(\alpha_{n}\right) \in \mathbb{R}^{\mathbb{N}}$ such that $\left(n \alpha_{n}\right) \in \ell^{s_{r q}}$ (note that the element $h_{j_{0}} \alpha_{1}$ is positioned at the $j_{0}$ th row and the $j_{0}$ th column of the matrix).

\section{Domination by Basis Operators and Representing Operators}

As a result of the active research in several branches of the harmonic analysis, a lot of information is known about weighted norm inequalities for classical operators on weighted Banach function spaces, mainly regarding weighted $L^{p}$ and Lorentz spaces. The bibliography on the subject is extremely broad; we refer the reader to [7] for the classical inequalities and to [2; 4] and the references therein for an updated review of the state of the art. We will use also some concrete results and ideas concerning weighted norm inequalities that can be found in the papers $[1$; 8; 13; 14].

We will show in what follows the characterization in terms of vector norm inequalities of what we call a representing operator for a Banach function space $X(\mu)$. This is essentially a modification of a basis operator $\mathcal{F}: L(\mu) \rightarrow \Lambda$ (for $L(\mu)$ being another Banach function space and $\Lambda$ a Banach space having an unconditional basis) that allows us to identify each function in $X(\mu)$ with some easy transformation of the basic coefficients of certain univocally associated function. Our motivation is given by the fact that, although the coefficients are not associated with a basis of the space $X(\mu)$, such kind of operator-which we will call 
a representing operator-allows us to find an easy representation of the functions of the space by means of some basic-type coefficients. If $\mathcal{F}$ is a basis operator, then we write $\left(\alpha_{i}(f)\right)_{i=1}^{\infty} \in \Lambda$ for the basic coefficients of a function $f$, that is, $\mathcal{F}(f)=\left(\alpha_{i}(f)\right)_{i=1}^{\infty}$.

Definition 6.1. Let $X(\mu)$ be a Banach function space over $\mu$, and let $\ell$ be a sequence space over the counting measure $c$ on $\mathbb{N}$. Let $\mathcal{B}$ be a Schauder basis of a Banach function space $L(\mu)$ and suppose that the basic coefficients of the functions of $L(\mu)$ are in a sequence space $\Lambda$ defined as the Banach lattice generated also by an unconditional basis of a Banach space. Consider an operator $T: X(\mu) \rightarrow \ell$. We will say that $T$ is a representing operator for $X(\mu)$ (with respect to $\mathcal{F}$ ) if it is an injective two-sides-diagonal transformation of the basis operator $\mathcal{F}$.

Thus, technically, a representing operator is an injective map such that there are a sequence $g=\left(g_{j}\right) \in \Lambda^{\ell}$ with $g_{j} \neq 0$ for all $j \in \mathbb{N}$ and a function $h \in X(\mu)^{L(\mu)}$, $h \neq 0 \mu$-a.e., such that for every $x \in X(\mu)$, the sequence $T(x)=\left(\beta(x)_{j}\right) \in \ell$ can be written as

$$
\beta(x)_{j}=P_{j} \circ T(x)=g_{j} \mathcal{F}(h x)_{j}=g_{j} \alpha_{j}(h x),
$$

where $P_{j}$ gives the $j$ th coordinate of $T(x)$; that is, for the elements $y \in h \cdot X(\mu) \subset$ $L(\mu)$, we have that

$$
\alpha_{j}(y)=\mathcal{F}(y)_{j}=g_{j}^{-1} \beta\left(h^{-1} y\right)_{j} .
$$

Equivalently, for each $x \in X(\mu)$, there is a sequence $\left(\beta_{j}\right) \in \ell$ such that

$$
x=T^{-1}\left(\left(\beta_{j}\right)\right)=h^{-1} \mathcal{F}^{-1}\left(\left(g_{j}^{-1} \beta_{j}\right)\right) .
$$

EXAMPLE 6.2. (i) An easy example of the introduced notion is the so-called generalized Fourier series. Consider $p=2$, an interval $I$ of the real line, the space $L^{2}(I)$ endowed with Lebesgue measure $d m$, and a weight function $w: I \rightarrow \mathbb{R}^{+}, w>0$. Note that the multiplication operator $M_{w^{1 / 2}}$ : $L^{2}(w d m) \rightarrow L^{2}(I)$ defines an isometry. Take a sequence of functions $\left(\phi_{n}\right)_{n}$ belonging to $L^{2}(w d m)$ and such that the associated sequence $\left(b_{n}\right)_{n}$, where $b_{n}=w^{1 / 2} \phi_{n}$ for all $n$, defines an orthonormal basis $\mathcal{B}$ in $L^{2}(I)$, that is, it is orthogonal, norm one, and complete. Note that this is equivalent to say that it defines an orthonormal basis in the weighted space $L^{2}(w d m)$. Consider the Fourier operator $\mathcal{F}_{\mathcal{B}}$ associated with the basis $\mathcal{B}$ of $L^{2}(I)$. Then the operator $T: L^{2}(w d m) \rightarrow \ell^{2}$ given by $T:=i d_{\ell^{2}} \circ \mathcal{F}_{\mathcal{B}} \circ M_{w^{1 / 2}}$ is a representing operator for $L^{2}(w d m)$.

Concrete examples of this situation are given by classical orthogonal bases of polynomials in weighted $L^{2}$-spaces. For example, for the trivial case of the weight equal to 1 and the space $L^{2}[-1,1]$, we can define the functions $\phi_{n}$ to be the Legendre polynomials, which are solutions to the SturmLiouville problem and define the corresponding Fourier-Legendre series. Other nontrivial cases, also for $I=(-1,1)$, are given by the weight functions 
$w(x)=\left(1-x^{2}\right)^{-1 / 2}(x)$ and $w(x)=\left(1-x^{2}\right)^{1 / 2}(x)$ and the Chebyshev polynomials of the first and second kinds, respectively. The Laguerre polynomials give another example for $I=(0, \infty)$ and the weight function $w(x)=e^{-x}$.

(ii) Take a function $0<h \in X(\mu)^{L^{2}(\mu)}$ and consider a sequence $0<\lambda=\left(\lambda_{i}\right) \in$ $\ell^{2}$. Let $c$ be the counting measure in $\mathbb{N}$. Consider the space $\ell^{1}(\lambda c)=\left\{\left(\tau_{i}\right)\right.$ : $\left.\left(\lambda_{i} \tau_{i}\right) \in \ell^{1}\right\}$ with the corresponding norm $\left\|\left(\tau_{i}\right)\right\|_{\ell^{1}(\lambda c)}=\sum\left|\lambda_{i} \tau_{i}\right|$. Then we have that $\ell^{2} \hookrightarrow \ell^{1}(\lambda c)$, and so the space of multiplication operators $\left(\ell^{2}\right)^{\ell^{1}(\lambda c)}$ is not trivial. A direct computation shows also that $\left(\ell^{2}\right)^{\ell^{1}(\lambda c)}=\ell^{2}\left(\lambda^{2} c\right)$. Then, for every $\tau=\left(\tau_{i}\right) \in \ell^{2}\left(\lambda^{2} c\right)$ with $\tau_{i} \neq 0$ for all $i \in \mathbb{N}$, we have that the operator $T: X(\mu) \rightarrow \ell^{1}(\lambda c)$ given by $T(\cdot)=\tau \mathcal{F}(h \cdot)$ is a representing operator for the space $X(\mu)$.

Let $J$ be a finite subset of $\mathbb{N}$, and write $P_{J}: \ell \rightarrow \ell$ for the standard projection on the subspace generated by the elements of $\mathcal{B}$ with subindexes in $J$. If $T: X(\mu) \rightarrow$ $\ell$ is an operator, then consider the net $\left\{P_{J} \circ T=: T_{J}: \mathbb{N} \supset J\right.$ finite $\}$, where the order is given by the inclusion of the set of subindexes, that is, $P_{J} \circ T \leq P_{J^{\prime}} \circ T$ if and only if $J \subset J^{\prime}$. By definition,

$$
T=\lim _{J} P_{J} \circ T
$$

as a pointwise limit. In what follows, we will characterize representing operators in terms of inequalities using this approximation procedure and a compactness argument. Thus, considering the basic (biorthogonal) functionals $b_{i}^{\prime} \in L(\mu)^{\prime}, i \in$ $\mathbb{N}$, associated with the basis of $L(\mu)$ that defines the Fourier operator that we are considering. Let $\left\{e_{j}: j \in \mathbb{N}\right\}$ be the unconditional basis of $\Lambda$ and $y_{i}^{\prime} \in \ell^{\prime}, i \in \mathbb{N}$.

Fix a function $h \in X(\mu)^{L(\mu)}$ and suppose that $\Lambda^{\ell}$ is nontrivial. Assume that the conditions are given to obtain that $\Lambda^{\ell}=\left(\ell^{\prime}\right)^{\Lambda^{\prime}}=\left(\Lambda \pi \ell^{\prime}\right)^{*}$. The domination inequality that must be considered in this case is given by the following expression:

$$
\begin{aligned}
\sum_{i=1}^{n} \int P_{J} \circ T\left(x_{i}\right) y_{i}^{\prime} d c & \leq\left\|\sum_{i=1}^{n}\left(\sum_{j \in J}\left\langle h x_{i}, b_{j}^{\prime}\right\rangle e_{j}\right) \cdot y_{i}^{\prime}\right\|_{\Lambda \pi \ell^{\prime}} \\
& =\sup _{g \in B_{\Lambda^{\ell}}}\left(\sum_{i=1}^{n} \sum_{j \in J}\left\langle h x_{i}, b_{j}^{\prime}\right\rangle\left\langle e_{j}, g y_{i}^{\prime}\right\rangle\right),
\end{aligned}
$$

that is, we are considering the sequence $\left(\sum_{i=1}^{n}\left\langle h x_{i}, b_{j}^{\prime}\right\rangle\left(y_{i}^{\prime}\right)_{j}\right)_{j \in J} \in \Lambda \pi \ell^{\prime}$ as the functional of the dual of $\Lambda^{\ell}$ given by

$$
\left(\sum_{i=1}^{n} \sum_{j \in J}\left\langle h x_{i}, b_{j}^{\prime}\right\rangle\left\langle e_{j}, \cdot y_{i}^{\prime}\right\rangle\right): \Lambda^{\ell} \rightarrow \mathbb{R} .
$$


After taking into account the particular descriptions of the elements of the spaces involved, we get the equivalent expression for the inequality

$$
\begin{aligned}
& \sum_{i=1}^{n} \sum_{j \in J} T\left(x_{i}\right)_{j}\left(y_{i}^{\prime}\right)_{j} \\
& \quad \leq \sup _{g \in B_{\Lambda^{\ell}}}\left(\sum_{i=1}^{n} \sum_{j \in J}\left\langle h x_{i}, b_{j}^{\prime}\right\rangle\left\langle e_{j},\left(g_{j}\left(y_{i}^{\prime}\right)_{j}\right)\right\rangle\right) \\
& \quad=\sup _{g \in B_{\Lambda^{\ell}}}\left(\sum_{i=1}^{n} \sum_{j \in J}\left\langle h x_{i}, b_{j}^{\prime}\right\rangle g_{j}\left(y_{i}^{\prime}\right)_{j}\right),
\end{aligned}
$$

and so the initial inequality is equivalent to the following one:

$$
\sum_{i=1}^{n} \sum_{j \in J} T\left(x_{i}\right)_{j}\left(y_{i}^{\prime}\right)_{j} \leq \sup _{g \in B_{\Lambda} \ell}\left(\sum_{i=1}^{n} \sum_{j \in J} \alpha_{j}\left(h x_{i}\right) g_{j}\left(y_{i}^{\prime}\right)_{j}\right),
$$

where $\alpha_{j}\left(h x_{i}\right)=\int h x_{i} b_{j}^{\prime} d \mu, j \in J$, are the $j$ th Fourier coefficients of the function $h x_{i}$ associated with the basis $\mathcal{B}$.

Thus the assumptions on the properties of $X(\mu)^{L(\mu)}$ and $\Lambda^{\ell}$ provide the following:

Theorem 6.3. Suppose that $\Lambda$ and $\ell$ satisfy that $\Lambda \pi \ell^{\prime}$ is saturated and $\left(\Lambda \pi \ell^{\prime}\right)^{*}=\Lambda^{\ell}$, and let $h$ be a measurable function such that $0<|h| \in X(\mu)^{L(\mu)}$. The following statements are equivalent for an operator $T: X(\mu) \rightarrow \ell$.

(i) For every finite set $J \subset \mathbb{N}$,

$$
\sum_{i=1}^{n} \sum_{j \in J} T\left(x_{i}\right)_{j}\left(y_{i}^{\prime}\right)_{j} \leq C \sup _{g \in B_{\Lambda} \ell}\left(\sum_{i=1}^{n} \sum_{j \in J} \alpha_{j}\left(h x_{i}\right) g_{j}\left(y_{i}^{\prime}\right)_{j}\right)
$$

for all $x_{1}, \ldots, x_{n} \in X(\mu)$ and $y_{1}^{\prime}, \ldots, y_{n}^{\prime} \in \ell^{\prime}$.

(ii) The map $T$ is a representing operator with respect to $\mathcal{F}$, that is, there is a sequence $g \in \Lambda^{\ell}$ such that $(T(x))_{j}=g_{j} \cdot \alpha_{j}(h x)$ for all $x \in X(\mu)$ and $j \in \mathbb{N}$. In other words, $T$ factors through $\mathcal{F}$ as

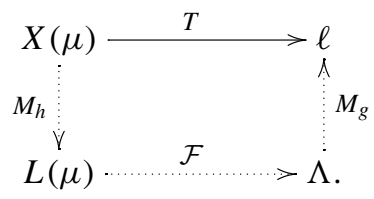

Proof. Let us see that (i) implies (ii). We can assume without loss of generality that $C=1$. Note that, as a consequence of Remark 3.3, the requirements on $\Lambda$ and $\ell$ provide the conditions on these spaces for applying Corollary 3.2. Indeed, as we explained in Section 2, $\Lambda \pi \ell^{\prime}$ saturated implies that $\left(\Lambda \pi \ell^{\prime}\right)^{\prime}=\Lambda^{\ell^{\prime \prime}}$, and this is also a saturated space. Since, by hypothesis,

$$
\left(\Lambda \pi \ell^{\prime}\right)^{\prime} \subset\left(\Lambda \pi \ell^{\prime}\right)^{*}=\Lambda^{\ell} \subset \Lambda^{\ell^{\prime \prime}}=\left(\Lambda \pi \ell^{\prime}\right)^{\prime},
$$


we have that $\left(\Lambda \pi \ell^{\prime}\right)^{\prime}=\left(\Lambda \pi \ell^{\prime}\right)^{*}$, and so by the characterization of order continuity we have that it is $\sigma$-order continuous. This, as explained in Remark 3.3, allows us to apply Corollary 3.2.

By the computations previous to the theorem, we obtain that for each finite set $J$, we have a norm one sequence $g_{J} \in \Lambda^{\ell}$ satisfying that

$$
P_{J} \circ T(x)=g_{J} \cdot P_{J} \circ \mathcal{F}(h x) .
$$

Consider the net $\mathcal{N}:=\left\{g_{J}: J \subset \mathbb{N}\right.$ finite $\}$, where the order is given by the inclusion of the finite sets used for the subindexes. We can assume without loss of generality that the support of each function $g_{J}$ is in $J$, that is, the coefficients $\left(g_{J}\right)_{k}$ of the sequence $g_{J}$ are 0 for $k \notin J$. Since all the functions of the net are in the unit ball, due to the product compatibility of the pair defined by $\Lambda$ and $\ell^{\prime}$, we have that the net is included in the weak* compact set $B_{\Lambda^{\ell}}$. Therefore it has a convergent subnet $\mathcal{N}_{0}$, that is, there is a sequence $g_{0} \in B_{\Lambda^{\ell}}$ such that

$$
\lim _{\eta \in \mathcal{N}_{0}} g_{\eta}=g_{0}
$$

in the weak* topology given by the dual pair $\left\langle\Lambda \pi \ell^{\prime}, \Lambda^{\ell}\right\rangle$.

Note now that for a fixed $x \in X(\mu)$, since we are assuming that $\ell$ has a unconditional basis with associated projections $P_{J}$, we have

$$
\lim _{J \in \mathcal{N} \text { finite }} P_{J} \circ T(x)=T(x) .
$$

Then

$$
T(x)=\lim _{J \in \mathcal{N} \text { finite }} P_{J} \circ T(x)=\lim _{\eta \in \mathcal{N}_{0}} P_{\eta} \circ T(x)=\lim _{\eta \in \mathcal{N}_{0}} g_{\eta} \cdot \mathcal{F}(h x)=g_{0} \cdot \mathcal{F}(h x) .
$$

This gives (ii) and finishes the proof, since the converse holds by a direct computation.

Let us provide an example. Consider again Example 6.2(ii) and recall that $\left(\ell^{2}\right)^{\ell^{1}(\lambda c)}=\ell^{2}\left(\lambda^{2} c\right)$. Theorem 6.3 gives that an injective operator $T: X(\mu) \rightarrow$ $\ell^{1}(\lambda c)$ is a representing operator by means of the Fourier operator if and only if for every finite set $J \subset \mathbb{N}$,

$$
\sum_{i=1}^{n} \sum_{j \in J} T\left(x_{i}\right)_{j}\left(y_{i}^{\prime}\right)_{j} \leq C \sup _{g \in B_{\ell^{2}\left(\lambda^{2} c\right)}}\left(\sum_{i=1}^{n} \sum_{j \in J} \alpha_{j}\left(h x_{i}\right) g_{j}\left(y_{i}^{\prime}\right)_{j}\right)
$$

for all $x_{1}, \ldots, x_{n} \in X(\mu)$ and $y_{1}^{\prime}, \ldots, y_{n}^{\prime} \in \ell^{1}(\lambda c)^{\prime}$.

REMARK 6.4. Let us gives some sufficient conditions for the product sequence space appearing in Theorem 6.3 to satisfy what is needed. Let $1<p<\infty$. The requirement on the pair $\ell^{p}$ and $\ell$ is

$$
\left(\ell^{p}\right)^{\ell}=\left(\ell^{\prime}\right)^{\ell^{p^{\prime}}}=\left(\ell^{p} \pi \ell^{\prime}\right)^{*} .
$$

For example, if $\ell^{\prime}$ is $p^{\prime}$-convex, then we have that $\ell^{p} \pi \ell^{\prime}$ is saturated and thus a Banach function space (see Proposition 2.2 in [16]). Moreover, the quoted result 
provides also the equality (under the assumption of saturation of the product)

$$
\left(\ell^{p} \pi \ell^{\prime}\right)^{\prime}=\left(\ell^{\prime} \pi \ell^{p}\right)^{\prime}=\left(\ell^{\prime}\right)^{\ell^{p^{\prime}}}=\left(\ell^{p}\right)^{\ell} .
$$

Consequently, if the product is order continuous, then we get the desired result. Conditions under which this space is order continuous are given in Corollary 5.3 in [6]. If the norm of the product is equivalent to

$$
\|\lambda\|_{\pi} \sim \inf \left\{\|\eta\|_{\ell^{p}} \cdot\|\gamma\|_{\ell^{\prime}}:|\lambda|=\eta \cdot \gamma, \eta \in \ell^{p}, \gamma \in \ell^{\prime}\right\}
$$

then the space is order continuous if $\ell^{\prime}$ is assumed to be order continuous (recall that $p>1$, and so $\ell^{p^{\prime}}$ is order continuous too). Besides these sufficient conditions, a complete characterization of order continuity in this case is given in [10, Cor. 1]. The coincidence with the previous formula for the product space works, for example, if $\ell$ is $p$-concave, since this implies that $\ell^{\prime}$ is $p^{\prime}$-convex, which, together with the $p$-convexity of $\ell^{p}$, provides the result. Concrete examples for $\ell^{p}$ spaces have been given in Example 6.2.

\section{Operators Associated to Trigonometric Series}

Relevant historical examples are the ones associated with the Fourier series and the corresponding Fourier coefficients. We finish the paper by explicitly writing the results presented previously in this setting. We write $\hat{x}(\cdot)$ for the $i$ th Fourier (real) coefficients of the function $x$ with indexes in the set $\mathbb{Z}$, writing the coefficients $a_{n}$ associated with cos functions as $\hat{x}(i)$ with positive $i$ and the coefficients $b_{n}$ for the functions $\sin$ as $\hat{x}(i)$ with negative $i$.

- Due to the Hausdorff-Young inequality, we know that for $1<p \leq 2$, the Fourier transform $\mathcal{F}_{p}: L^{p}[-\pi, \pi] \rightarrow \ell^{p^{\prime}}$ that assigns to each function the sequence of its Fourier coefficients is well-defined and continuous. The Fourier transform is defined as $\mathcal{F}_{2}: L^{2} \rightarrow \ell^{2}$. Suppose that we want to check if a particular operator $\mathcal{G}_{2}: L^{2}[-\pi, \pi] \rightarrow \ell^{2}$ can be extended to $L^{p}[-\pi, \pi]$ through $\mathcal{F}_{p}$; that is, if there is a factorization for $\mathcal{G}_{2}$ as

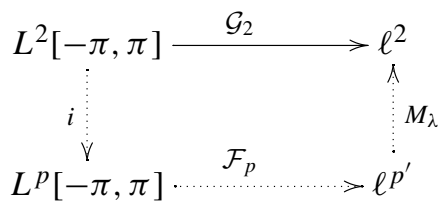

for the operator $\mathcal{G}_{2}$ for some multiplication operator given by a sequence $\lambda$.

We have shown that this is equivalent to the following inequalities for the operator $\mathcal{G}_{2}$ : For all $x_{1}, \ldots, x_{n} \in L^{2}[-\pi, \pi]$ and $\lambda_{i} \in \ell^{2}$,

$$
\begin{aligned}
& \sum_{k=1}^{\infty} \sum_{i=1}^{n}\left(\mathcal{G}_{2}\left(x_{i}\right)\right)_{k}\left(\lambda_{i}\right)_{k} \\
& \quad \leq C\left\|\left(\sum_{i=1}^{n} \hat{x}_{i}(k)\left(\lambda_{i}\right)_{k}\right)\right\|_{\ell^{r}}=C\left(\sum_{k=1}^{\infty}\left|\sum_{i=1}^{n} \hat{x}_{i}(k)\left(\lambda_{i}\right)_{k}\right|^{r}\right)^{1 / r} .
\end{aligned}
$$


- For $1<p \leq 2$ again, Kellogg proved an improvement of the Hausdorff-Young inequality, which assures that the corresponding Fourier coefficients of the functions in $L^{p}$ can be found in the smaller mixed norm space $L^{p^{\prime}, 2} \subset \ell^{p^{\prime}}$. Fix $1 \leq p, q \leq \infty$. The mixed norm sequence space $L^{p, q}$ was defined in [8] as the space of sequences $\lambda=\left(\lambda_{k}\right)_{-\infty}^{\infty}$ such that

$$
\|\lambda\|=\left(\sum_{m=-\infty}^{\infty}\left(\sum_{k \in I(m)}\left|\lambda_{k}\right|^{p}\right)^{q / p}\right)^{1 / q}<\infty
$$

where $I(m)=\left\{k \in \mathbb{Z}: 2^{m-1} \leq k \leq 2^{m}\right\}$ if $m>0, I(0)=\{0\}$, and $I(m)=\{k \in$ $\left.\mathbb{Z}:-2^{-m} \leq k \leq-2^{-m-1}\right\}$ if $m<0$. It is easy to see that $L^{p^{\prime}, 2} \subset \ell^{p^{\prime}}$, and so we have a factorization for the Fourier map as

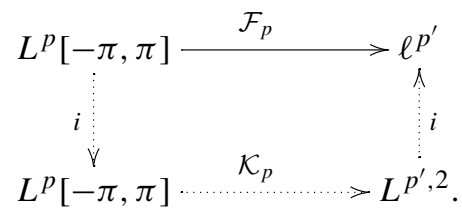

In Theorem 1 of [8], it is proved that the space of multiplication operators (multipliers) from $L^{p^{\prime}, 2}$ to $\ell^{p^{\prime}}$ can in fact be identified with $\ell^{\infty}$. Consequently, our results imply that for every finite set $J \subset \mathbb{Z}$, the inequality

$$
\sum_{i=1}^{n} \sum_{j \in J}\left(\mathcal{F}_{p} x_{i}\right)_{j}\left(\lambda_{i}^{\prime}\right)_{j} \leq C \sup _{g \in B_{\ell} \infty}\left(\sum_{i=1}^{n} \sum_{j \in J} \hat{x}_{i}(j) g_{j}\left(\lambda_{i}^{\prime}\right)_{j}\right)
$$

holds for all $x_{1}, \ldots, x_{n} \in L^{p}[-\pi, \pi]$ and $\lambda_{1}^{\prime}, \ldots, \lambda_{n}^{\prime} \in \ell^{p}$, which is obvious. However, note that this is essentially a characterization, since any other operator $\mathcal{G}_{p}$ from $L^{p}$ having values in a sequence space $\ell$ such that $\left(L^{p^{\prime}, 2}\right)^{\ell}=\ell^{\infty}$ and satisfying these inequalities has to be necessarily of the form $g \cdot \mathcal{K}_{p}$ for a certain sequence $g \in \ell^{\infty}$.

- The Hardy-Littlewood inequality, also for $1<p \leq 2$, provides an example of an operator $\mathcal{H}_{p}$ sending the Fourier coefficients of the functions in $L^{p}$ to a weighted $\ell^{p}$ space. For $1<p<2$, consider the weighted sequence space $\ell^{p}(W)$, where the weight $W$ is given by $W=\left(W_{n}\right)=\left(1 /(n+1)^{2-p}\right)$. The Hardy-Littlewood inequality can be understood as the fact that the Fourier operator can be defined as $\mathcal{H}_{p}: L^{p}[-\pi, \pi] \rightarrow \ell^{p}(W)$ (see [1, S. 2], in particular, Theorem B). Note that the multiplication operator $M_{\gamma}: \ell^{p}(W) \rightarrow \ell^{p}$ given by the sequence $\gamma=\left(\left(1 /(n+1)^{\frac{2-p}{p}}\right)\right.$ defines an isometry. Therefore the factorization scheme

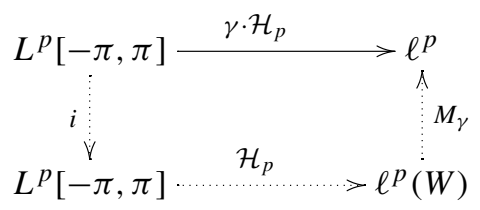


provides another example of the situation we are describing. Indeed, for every multiplication operator $M_{\tau}$ for $\tau \in\left(\ell^{p}(W)\right)^{\ell^{p}}$, we can give an operator $\tau \cdot \mathcal{H}_{p}$ satisfying this factorization. Our results implies that the class of all these operators is characterized in the following way: if $T: L^{p}[-\pi, \pi] \rightarrow \ell^{p}$ satisfies the inequalities

$$
\sum_{i=1}^{n} \sum_{j \in J}\left(T\left(x_{i}\right)\right)_{j}\left(\lambda_{i}^{\prime}\right)_{j} \leq C \sup _{g \in B_{(\ell p(W))^{\ell p}}}\left(\sum_{i=1}^{n} \sum_{j \in J} \hat{x}_{i}(j) g_{j}\left(\lambda_{i}^{\prime}\right)_{j}\right)
$$

for each finite subset $J \subset \mathbb{Z}$ and all $x_{1}, \ldots, x_{n} \in L^{p}[-2 \pi, 2 \pi]$ and $\lambda_{1}^{\prime}, \ldots, \lambda_{n}^{\prime} \in$ $\ell^{p^{\prime}}$, then it has a factorization as the one above for a certain $\tau \in\left(\ell^{p}(W)\right)^{\ell^{p}}$.

- Let us recall Example 6.2(i). A representing operator $T: L^{2}(w d m) \rightarrow \ell^{2}$ associated with a weight function $w$ and an orthogonal basis $\mathcal{B}$ with respect to the corresponding weight function was considered. It allowed a factorization as

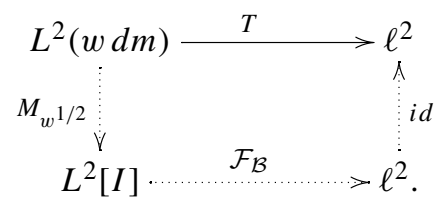

The corresponding vector norm inequality characterizing this factorization is

$$
\begin{aligned}
\sum_{i=1}^{n} \sum_{j \in J}\left(T\left(x_{i}\right)\right)_{j}\left(\lambda_{i}^{\prime}\right)_{j} & \leq C\left\|\sum_{i=1}^{n} \sum_{j \in J}\left(T\left(x_{i}\right)\right)_{j}\left(\lambda_{i}^{\prime}\right)_{j}\right\|_{\ell^{1}} \\
& =C \sum_{j \in J}\left|\sum_{i=1}^{n}\left(\mathcal{F}_{\mathcal{B}}\left(w^{1 / 2} x_{i}\right)\right)_{j}\left(\lambda_{i}^{\prime}\right)_{j}\right|
\end{aligned}
$$

for a given constant $C>0$, for each finite subset $J \subset \mathbb{N}$, and all $x_{1}, \ldots, x_{n} \in$ $L^{2}(w d m)$ and $\lambda_{1}^{\prime}, \ldots, \lambda_{n}^{\prime} \in \ell^{2}$.

ACKNOWLEDGMENT. We thank the anonymous referees for helpful comments, which improved the presentation.

\section{References}

[1] J. M. Ash, S. Tikhonov, and J. Tung, Wiener's positive Fourier coefficients theorem in variants of $L^{p}$ spaces, Michigan Math. J. 59 (2010), no. 1, 143-151.

[2] J. J. Benedetto and H. P. Heinig, Weighted Fourier inequalities: new proofs and generalizations, J. Fourier Anal. Appl. 9 (2003), no. 1, 1-37.

[3] J. M. Calabuig, O. Delgado, and E. A. Sánchez Pérez, Generalized perfect spaces, Indag. Math. (N.S.) 19 (2008), 359-378.

[4] D. Cruz-Uribe, J. M. Martell, and C. Pérez, Sharp weighted estimates for classical operators, Adv. Math. 229 (2012), no. 1, 408-441.

[5] O. Delgado and E. A. Sánchez Pérez, Summability properties for multiplication operators on Banach function spaces, Integral Equations Operator Theory 66 (2010), 197-214. 
[6] Strong factorizations between couples of operators on Banach function spaces, J. Convex Anal. 20 (2013), 599-616.

[7] G. H. Hardy, J. E. Littlewood, and G. Pólya, Inequalities, Cambridge University Press, 1934.

[8] C. N. Kellogg, An extension of the Hausdorff-Young theorem, Michigan Math. J. 18 (1971), 121-127.

[9] P. Kolwicz, K. Leśnik, and L. Maligranda, Pointwise multipliers of CalderónLozanovskii spaces, Math. Nachr. 286 (2013), no. 8, 876-907.

[10] _ Pointwise products of some Banach function spaces and factorization, J. Funct. Anal. 266 (2014), no. 2, 616-659.

[11] J. Lindenstrauss and L. Tzafriri, Classical Banach spaces, vol. II, Springer-Verlag, Berlin, 1979.

[12] L. Maligranda and L. E. Persson, Generalized duality of some Banach function spaces, Indag. Math. (N.S.) 51 (1989), 323-338.

[13] H. Mhaskar and S. Tikhonov, Wiener type theorems for Jacobi series with nonnegative coefficients, Proc. Amer. Math. Soc. 140 (2012), no. 3, 977-986.

[14] C. Pérez, Sharp $L^{p}$-weighted Sobolev inequalities, Ann. Inst. Fourier (Grenoble) 45 (1995), no. 3, 809-824.

[15] A. Pietsch, Operator ideals, North Holland, Amsterdam, 1980.

[16] E. A. Sánchez Pérez, Factorization theorems for multiplication operators on Banach function spaces, Integral Equations Operator Theory 80 (2014), no. 1, 117-135.

[17] A. R. Schep, Products and factors of Banach function spaces, Positivity 14 (2010), no. 2, 301-319.

[18] A. C. Zaanen, Integration, second edition, North Holland, Amsterdam, 1967.

O. Delgado

Departamento de Matemática Aplicada I

E. T. S. de Ingeniería de Edificación

Universidad de Sevilla

Avenida de Reina Mercedes

4 A, Sevilla 41012

Spain

olvido@us.es

E. A. Sánchez Pérez

Instituto Universitario de Matemática

Pura y Aplicada

Universitat Politècnica de València

Camino de Vera s/n

46022 Valencia

Spain
M. Mastyło

Faculty of Mathematics and Computer Science

Adam Mickiewicz University in Poznań

Umultowska 87

61-614 Poznań

Poland 